\title{
Condensation of $\alpha$-Aroylketene Dithioacetals and 2-Hydroxyarylaldehydes Results in Facile Synthesis of a Combinatorial Library of 3-Aroyl Coumarins
}

\author{
H. Surya Prakash Rao* and S. Sivakumar \\ Department of Chemistry \\ Pondicherry University \\ Pondicherry - 605014
}

1. General

\section{SUPPORTING INFORMATION}

\section{2. ${ }^{1} \mathbf{H \&}{ }^{13} \mathrm{C}$ NMR spectrum}

2.1. $300 \mathrm{MHz}\left(\mathrm{CDCl}_{3}\right){ }^{1} \mathrm{H} \mathrm{NMR}$ spectrum of

3,3-bis(methylsulfanyl)-1-(1-naphthyl)-2-propen-1-one $7 \quad \mathrm{~S}_{3}$

2.2. $75 \mathrm{MHz}\left(\mathrm{CDCl}_{3}\right){ }^{13} \mathrm{C} \mathrm{NMR}$ spectrum of

3,3-bis(methylsulfanyl)-1-(1-naphthyl)-2-propen-1-one $7 \quad \mathrm{~S}_{3}$

2.3. $300 \mathrm{MHz}\left(\mathrm{CDCl}_{3}\right){ }^{1} \mathrm{H} \mathrm{NMR}$ spectrum of

3,3-bis(methylsulfanyl)-1-(2-naphthyl)-2-propen-1-one $9 \quad \mathrm{~S}_{4}$

2.4. $75 \mathrm{MHz}\left(\mathrm{CDCl}_{3}\right){ }^{13} \mathrm{C} \mathrm{NMR}$ spectrum of

3,3-bis(methylsulfanyl)-1-(2-naphthyl)-2-propen-1-one $9 \quad \mathrm{~S}_{4}$

2.5. $300 \mathrm{MHz}\left(\mathrm{CDCl}_{3}\right){ }^{1} \mathrm{H} \mathrm{NMR}$ spectrum of

1-biphenyl-4-yl-3,3-bis-methylsulfanylprop-2-en-1-one $11 \quad \mathrm{~S}_{5}$

2.6. $75 \mathrm{MHz}\left(\mathrm{CDCl}_{3}\right){ }^{13} \mathrm{C} \mathrm{NMR}$ spectrum of

1-biphenyl-4-yl-3,3-bis-methylsulfanylprop-2-en-1-one $11 \quad \mathrm{~S}_{5}$

2.7. $300 \mathrm{MHz}\left(\mathrm{CDCl}_{3}\right){ }^{1} \mathrm{H} \mathrm{NMR}$ spectrum of

3,3-bis(methylsulfanyl)-1- $\eta^{5}$-ferrocenyl-2-propen-1-one $\mathbf{1 3} \quad \mathrm{S}_{6}$

2.8. $75 \mathrm{MHz}\left(\mathrm{CDCl}_{3}\right){ }^{1} \mathrm{H} \mathrm{NMR}$ spectrum of

3,3-bis(methylsulfanyl)-1- $\eta^{5}$-ferrocenyl-2-propen-1-one $\mathbf{1 3} \quad \mathrm{S}_{6}$

2.9. $300 \mathrm{MHz}\left(\mathrm{CDCl}_{3}\right){ }^{1} \mathrm{H} \mathrm{NMR}$ spectrum of

6-methyl-3-(4-methylbenzoyl)-2H-2-chromenone 4e $\quad \mathrm{S}_{7}$

2.10. $75 \mathrm{MHz}\left(\mathrm{CDCl}_{3}\right){ }^{13} \mathrm{C} \mathrm{NMR}$ spectrum of

6-methyl-3-(4-methylbenzoyl)-2 $\mathrm{H}$-2-chromenone 4e $\quad \mathrm{S}_{7}$

2.11. $300 \mathrm{MHz}\left(\mathrm{CDCl}_{3}\right){ }^{1} \mathrm{H} \mathrm{NMR}$ spectrum of

3-(4-Chlorobenzoyl)-6-methyl-2H-2-chromenone $\mathbf{4 f} \quad \mathrm{S}_{8}$

2.12. $75 \mathrm{MHz}\left(\mathrm{CDCl}_{3}\right){ }^{13} \mathrm{C} \mathrm{NMR}$ spectrum of

3-(4-Chlorobenzoyl)-6-methyl-2H-2-chromenone $\mathbf{4 f} \quad \mathrm{S}_{8}$

2.13. $400 \mathrm{MHz}\left(\mathrm{CDCl}_{3}\right){ }^{1} \mathrm{H}$ NMR spectrum of

6-methoxy-3-(4-methylbenzoyl)-2H-2-chromenone 4 h $\quad \mathrm{S}_{9}$

2.14. $100 \mathrm{MHz}\left(\mathrm{CDCl}_{3}\right){ }^{13} \mathrm{C} \mathrm{NMR}$ spectrum of

6-methoxy-3-(4-methylbenzoyl)-2H-2-chromenone 4 h $\quad \mathrm{S}_{9}$

2.15. $300 \mathrm{MHz}\left(\mathrm{CDCl}_{3}+\mathrm{DMSO}\right){ }^{1} \mathrm{H} \mathrm{NMR}$ spectrum of

3-(4-Chlorobenzoyl)-6-methoxy-2H-2-chromenone 4i $\quad \mathrm{S}_{10}$

2.16. $75 \mathrm{MHz}\left(\mathrm{CDCl}_{3}+\mathrm{DMSO}\right){ }^{13} \mathrm{C}$ NMR spectrum of 
3-(4-Chlorobenzoyl)-6-methoxy-2H-2-chromenone 4i $\quad \mathrm{S}_{10}$

2.17. $300 \mathrm{MHz}\left(\mathrm{CDCl}_{3}\right){ }^{1} \mathrm{H}$ NMR spectrum of

6-chloro-3-(4-methylbenzoyl)-2 $\mathrm{H}$-2-chromenone 4k $\quad \mathrm{S}_{11}$

2.18. $75 \mathrm{MHz}\left(\mathrm{CDCl}_{3}\right){ }^{13} \mathrm{C}$ spectrum of

6-chloro-3-(4-methylbenzoyl)-2 $H$-2-chromenone 4k $\quad \mathrm{S}_{11}$

2.19. $300 \mathrm{MHz}\left(\mathrm{CDCl}_{3}\right){ }^{1} \mathrm{H}$ NMR spectrum of

6-Chloro-3-(4-chlorobenzoyl)-2H-2-chromenone $41 \quad \mathrm{~S}_{12}$

2.20. $75 \mathrm{MHz}\left(\mathrm{CDCl}_{3}\right){ }^{13} \mathrm{C} \mathrm{NMR}$ spectrum of

6-chloro-3-(4-chlorobenzoyl)-2H-2-chromenone $41 \quad \mathrm{~S}_{12}$

2.21. $400 \mathrm{MHz}\left(\mathrm{CDCl}_{3}\right){ }^{1} \mathrm{H} \mathrm{NMR}$ spectrum of

3-(1-naphthylcarbonyl)-2H-2-chromenone $8 \quad \mathrm{~S}_{13}$

2.22. $100 \mathrm{MHz}\left(\mathrm{CDCl}_{3}\right){ }^{13} \mathrm{C} \mathrm{NMR}$ spectrum of

3-(1-naphthylcarbonyl)-2H-2-chromenone $8 \quad \mathrm{~S}_{13}$

2.23. $60 \mathrm{MHz}\left(\mathrm{CDCl}_{3}: \mathrm{CCl}_{4}\right){ }^{1} \mathrm{H} \mathrm{NMR}$ spectrum of

3-(2-naphthylcarbonyl)-2H-2-chromenone $\mathbf{1 0} \quad \mathrm{S}_{14}$

2.24. $100 \mathrm{MHz}\left(\mathrm{CDCl}_{3}\right.$ :DMSO-D ${ }_{6}{ }^{13} \mathrm{C}$ NMR spectrum of

3-(2-naphthylcarbonyl)-2H-2-chromenone $\mathbf{1 0} \quad \mathrm{S}_{14}$

2.25. $300 \mathrm{MHz}\left(\mathrm{CDCl}_{3}\right){ }^{1} \mathrm{H}$ NMR spectrum of

3-(biphenyl-4-yl-carbonyl)-2H-2-chromenone $12 \quad \mathrm{~S}_{15}$

2.26. $75 \mathrm{MHz}\left(\mathrm{CDCl}_{3}\right){ }^{13} \mathrm{C} \mathrm{NMR}$ spectrum of

3-(biphenyl-4-yl-carbonyl)-2H-2-chromenone $12 \quad \mathrm{~S}_{15}$

2.27. $400 \mathrm{MHz}\left(\mathrm{CDCl}_{3}\right){ }^{1} \mathrm{H}$ NMR spectrum of

3- $\eta^{5}$-ferrocenoyl- $2 \mathrm{H}$-2-chromenone 14

2.28. $100 \mathrm{MHz}\left(\mathrm{CDCl}_{3}\right){ }^{13} \mathrm{C} \mathrm{NMR} \mathrm{spectrum} \mathrm{of}$

$3-\eta^{5}$-ferrocenoyl-2H-2-chromenone 14

$\mathrm{S}_{16}$

$\mathrm{S}_{16}$

\section{General}

The progression of all the reactions was monitored by TLC using hexanes $\left(60-80{ }^{\circ} \mathrm{C}\right.$ boiling mixture) / ethyl acetate mixture as eluent. Column chromatography was carried on silica gel $\left(100-200\right.$ mesh) using increasing percentage of ethyl acetate in hexanes. The ${ }^{1} \mathrm{H}$ NMR (400 $\mathrm{MHz}$ or $300 \mathrm{MHz}$ or $60 \mathrm{MHz})$ and ${ }^{13} \mathrm{C} \mathrm{NMR} \mathrm{(100} \mathrm{MHz} \mathrm{or} 75 \mathrm{MHz}$ ) and DEPT spectra were recorded in $\mathrm{CDCl}_{3}$ or $\mathrm{CDCl}_{3}+\operatorname{DMSO}(5: 1)$ with TMS (0 ppm) as internal standard. The IR spectra were recorded as KBr solutions in a FT-IR (ZnSe optics) spectrometer. UV spectra were recorded using a ratio-beam spectrometer. Melting points were recorded using open-ended capillary tubes and are uncorrected. The methyl ketones, 4-phenylacetophenone and 
acetylferrocene 15 were prepared according to published procecdure. ${ }^{1}$ Hitherto known AKDTAs were prepared according to literature methods. ${ }^{2}$

\section{References:}

1. Furniss, B. S.; Hannaford, A. J.; Smith, P. W. G.; Tatchell, A. R. Vogel's Text Book of Practical Organic Chemistry, V Ed. 1989, Pearson Education, pp 1013-1014.

2. (a) Thuillier, A.; Vialle, J. Bull. Soc. Chim. Fr. 1959, 1398-1401; (b) Thuillier, A.; Vialle. J. Bull. Soc. Chim. Fr. 1962, 2194-2198; (c) Kumar, A.; Ila, H.; Junjappa. H. J. Chem. Res (S). 1979, 268-269; (d) Potts, K. T.; Cipullo, M. J.; Ralli, P.; Theodoridis, G. J. Org. Chem. 1982, 47, 3027-3038; (e) Asokan, C.V.; Bhattacharjee, S.; Ila, H.; Junjappa, H. Synthesis, 1988, 163-165; (f) Asokan, C.V.; Balu, M. P.; Ila, H.; Junjappa, H. Synthesis, 1988, 727-729. 

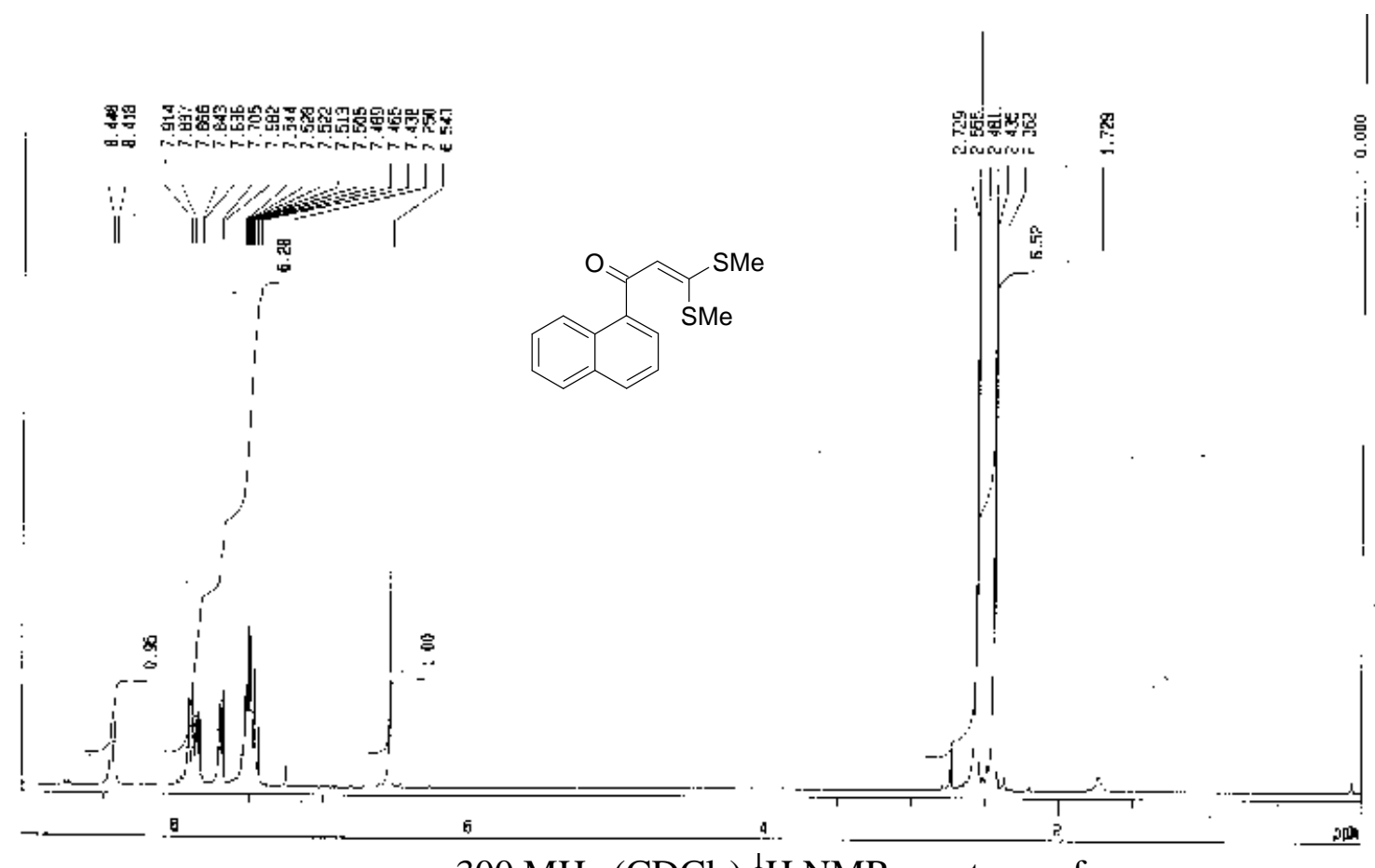

$300 \mathrm{MHz}\left(\mathrm{CDCl}_{3}\right){ }^{1} \mathrm{H}$ NMR spectrum of

3,3-bis(methylsulfanyl)-1-(1-naphthyl)-2-propen-1-one 7

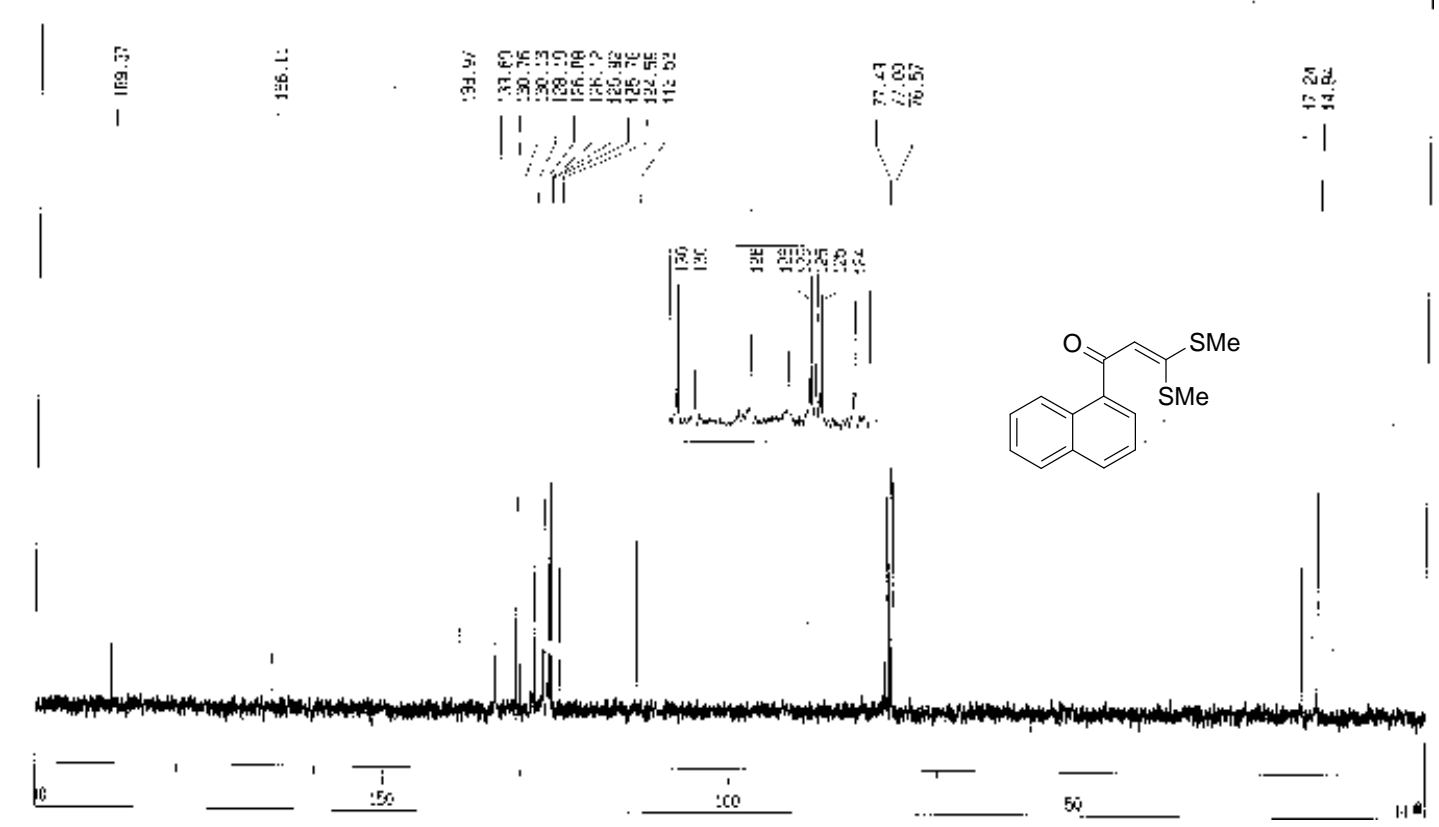

$75 \mathrm{MHz}\left(\mathrm{CDCl}_{3}\right){ }^{13} \mathrm{C}$ NMR spectrum of

3,3-bis(methylsulfanyl)-1-(1-naphthyl)-2-propen-1-one 7 

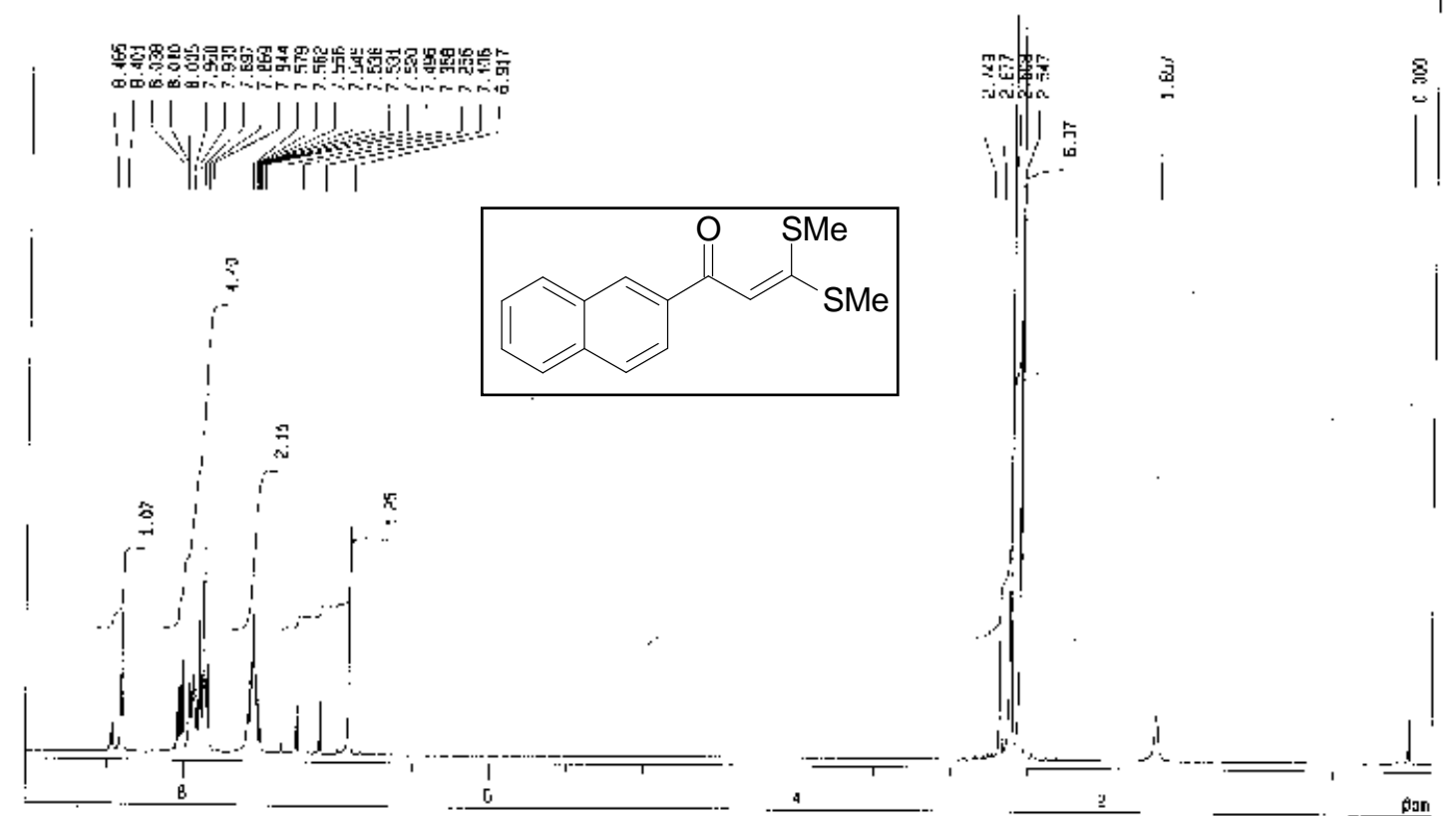

$300 \mathrm{MHz}\left(\mathrm{CDCl}_{3}\right){ }^{1} \mathrm{H}$ NMR spectrum of

3,3-bis(methylsulfanyl)-1-(2-naphthyl)-2-propen-1-one 9

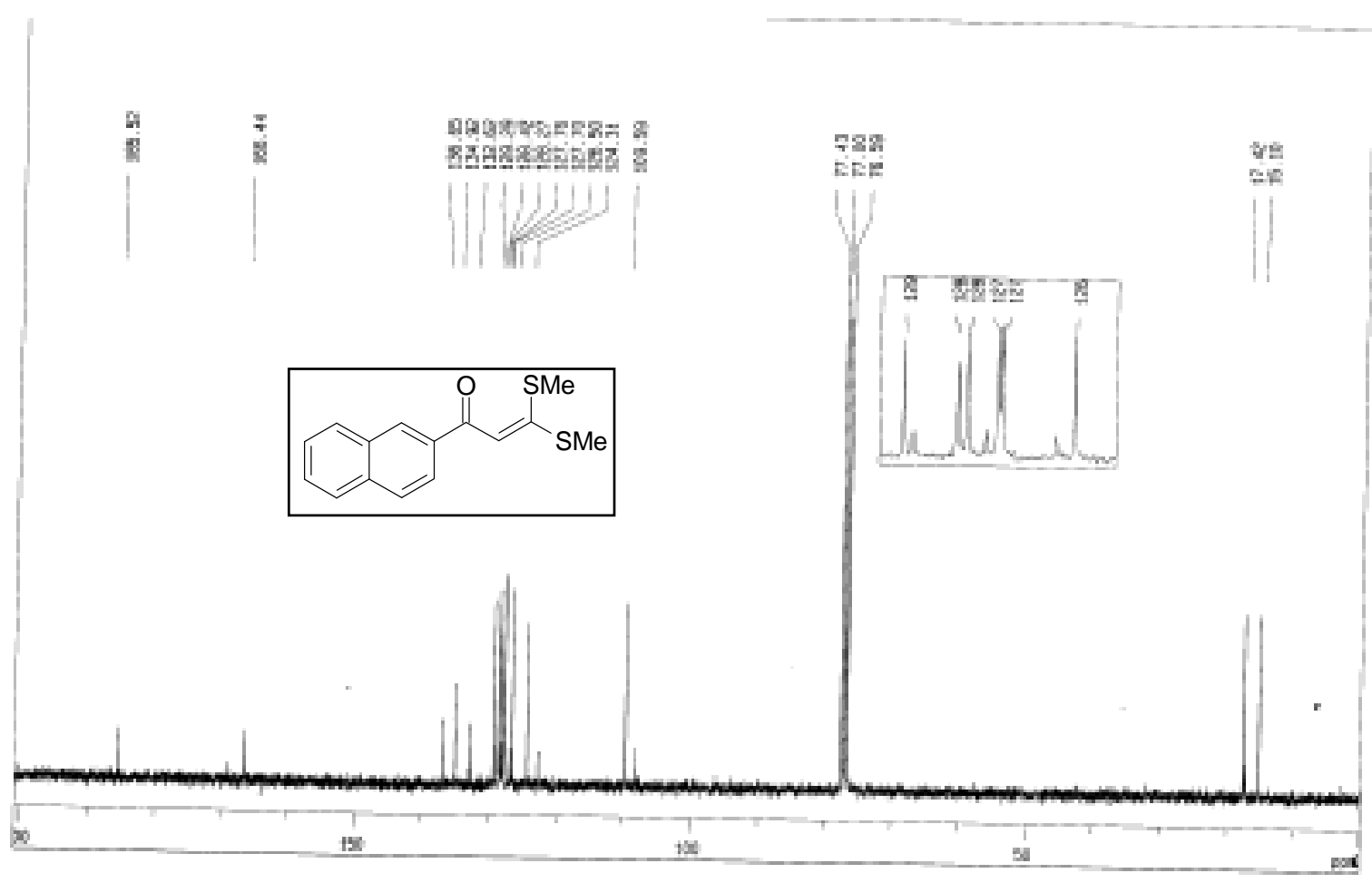

$75 \mathrm{MHz}\left(\mathrm{CDCl}_{3}\right){ }^{13} \mathrm{C}$ NMR spectrum of

3,3-bis(methylsulfanyl)-1-(2-naphthyl)-2-propen-1-one 9 
$-S_{6}-$

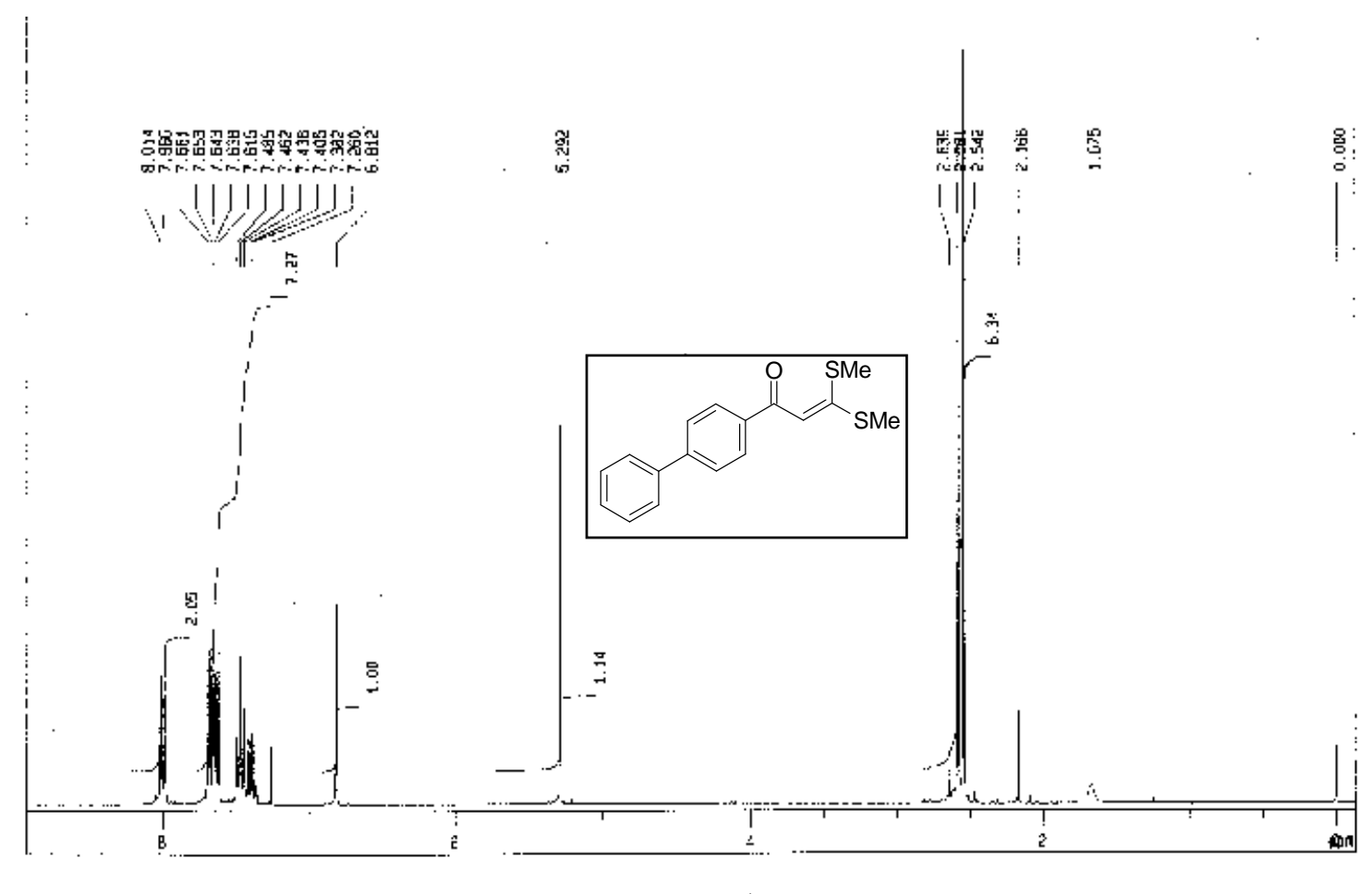

$300 \mathrm{MHz}\left(\mathrm{CDCl}_{3}\right)^{1} \mathrm{H}$ NMR spectrum of 1-biphenyl-4-yl-3,3-bismethylsulfanyl-2-propen-1-one 11

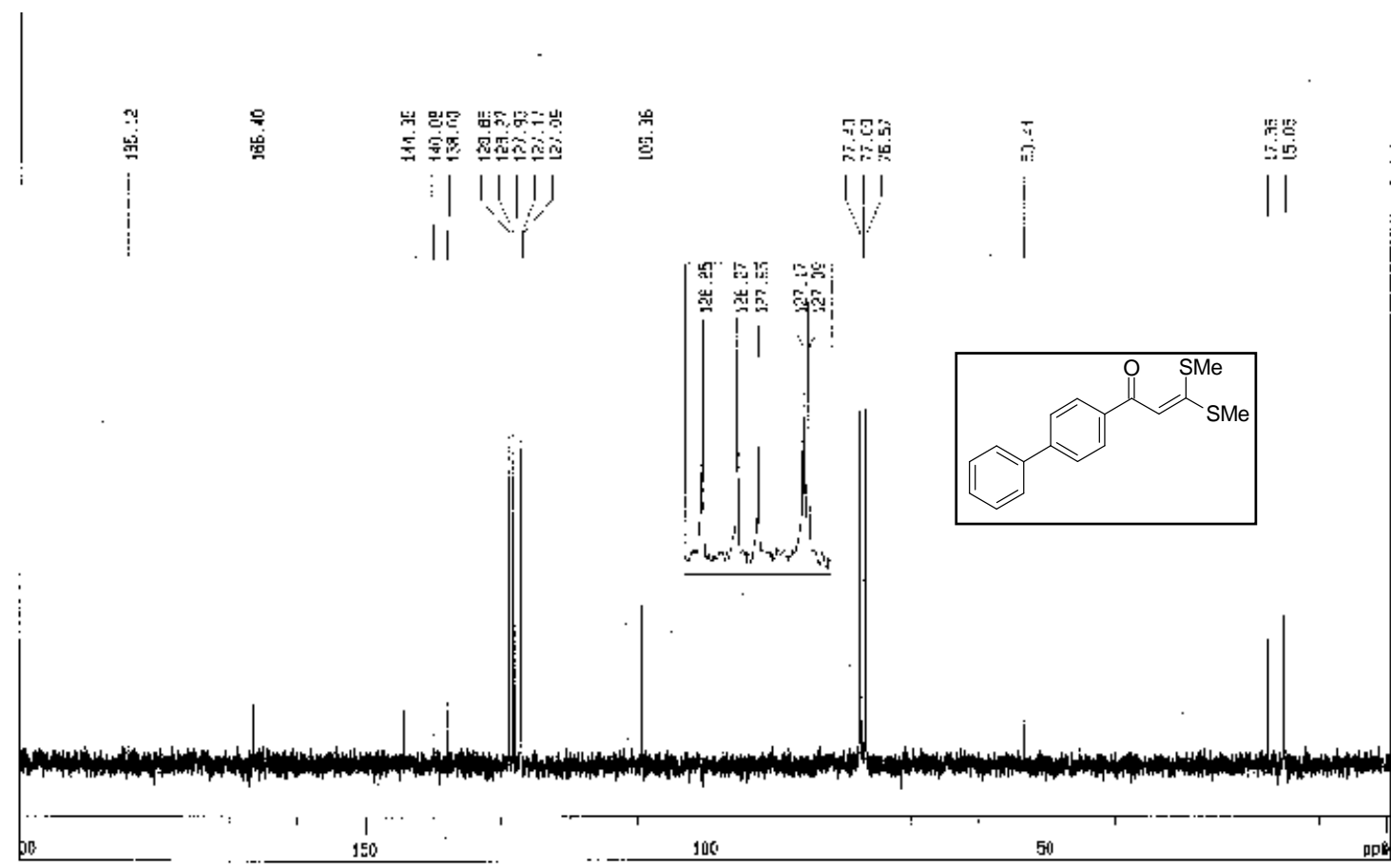

$75 \mathrm{MHz}\left(\mathrm{CDCl}_{3}\right){ }^{13} \mathrm{C}$ NMR spectrum of

1-biphenyl-4-yl-3,3-bismethylsulfanyl-2-propen-1-one 11 

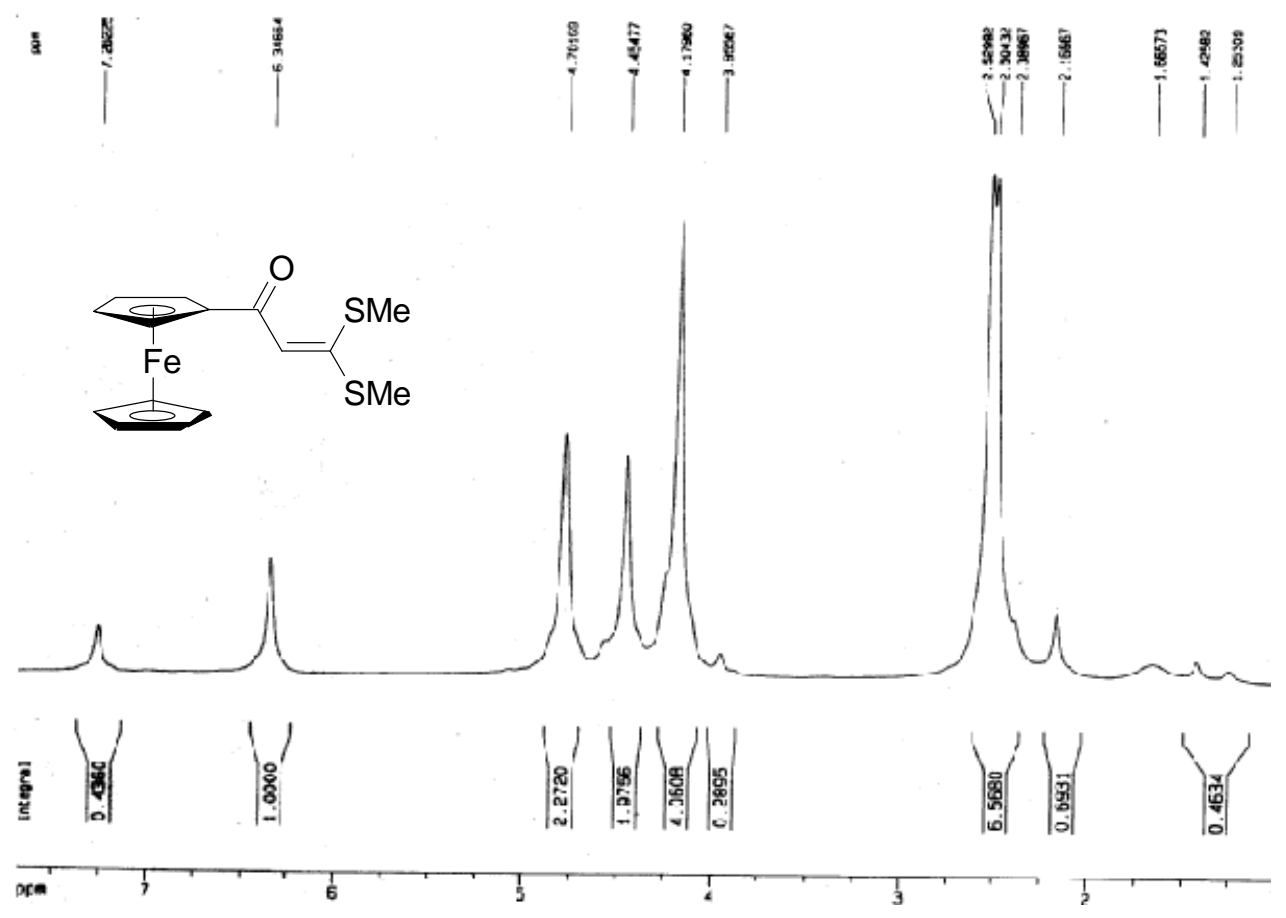

$300 \mathrm{MHz}\left(\mathrm{CDCl}_{3}\right){ }^{1} \mathrm{H} \mathrm{NMR}$ spectrum of

3,3-bis(methylsulfanyl)-1- $\eta^{5}$-ferrocenyl-2-propen-1-one $\mathbf{1 3}$

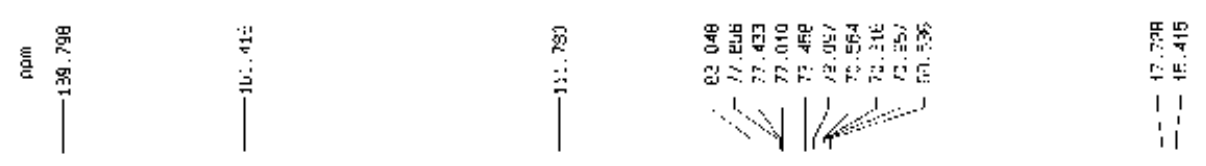

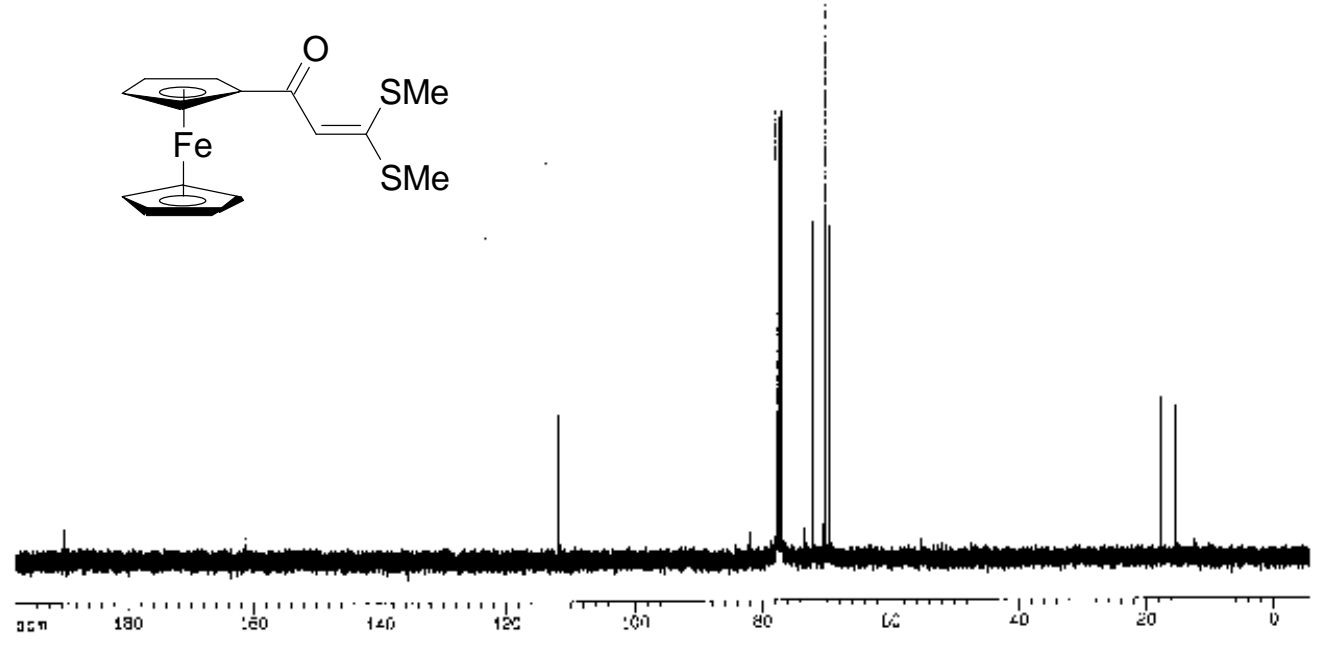

$75 \mathrm{MHz}\left(\mathrm{CDCl}_{3}\right){ }^{13} \mathrm{C}$ NMR spectrum of 3,3-bis(methylsulfanyl)-1- $\eta^{5}$-ferrocenyl-2-propen-1-one $\mathbf{1 3}$ 

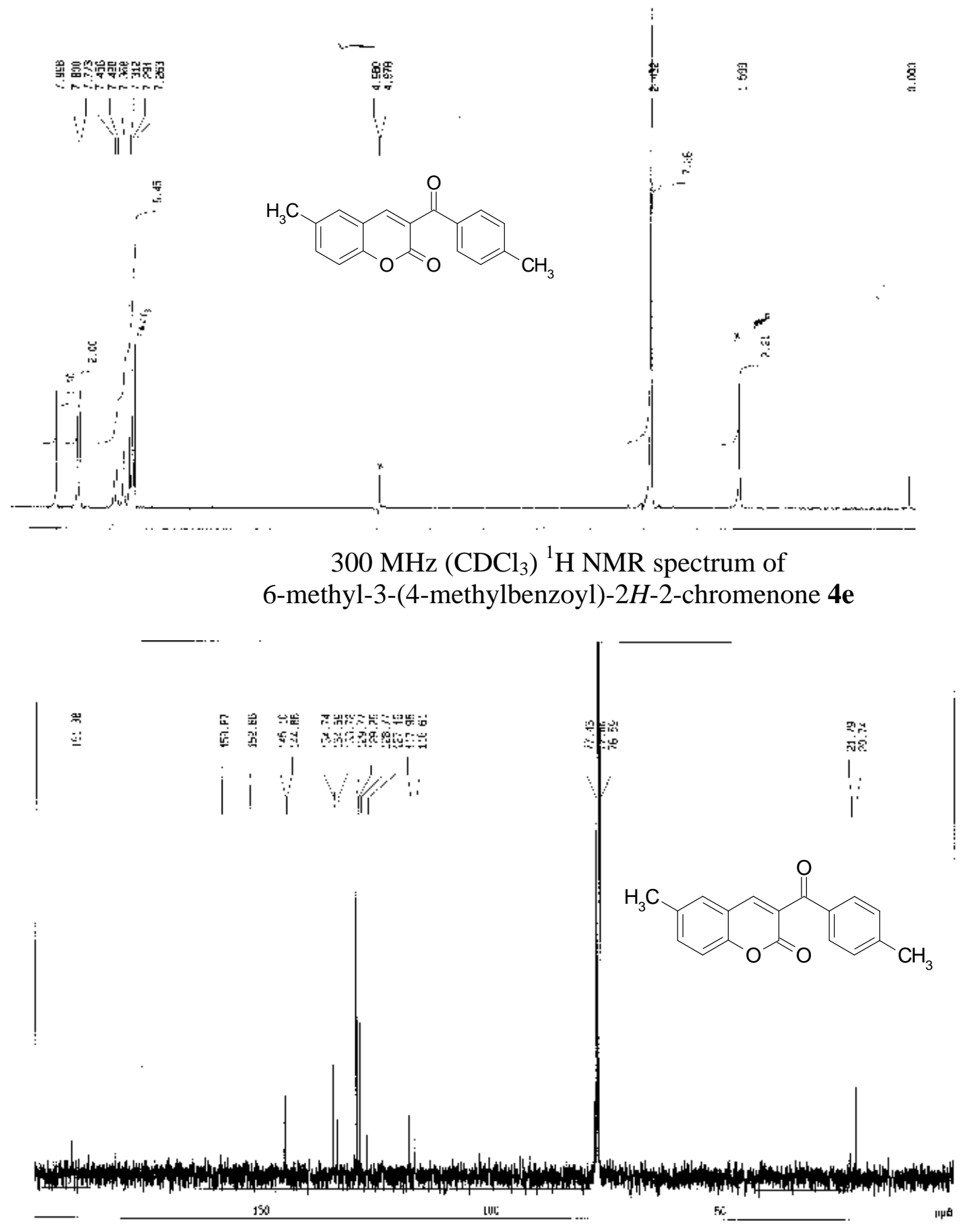

$75 \mathrm{MHz}\left(\mathrm{CDCl}_{3}\right){ }^{13} \mathrm{C}$ NMR spectrum of 6-methyl-3-(4-methylbenzoyl)-2H-2-chromenone 4e 


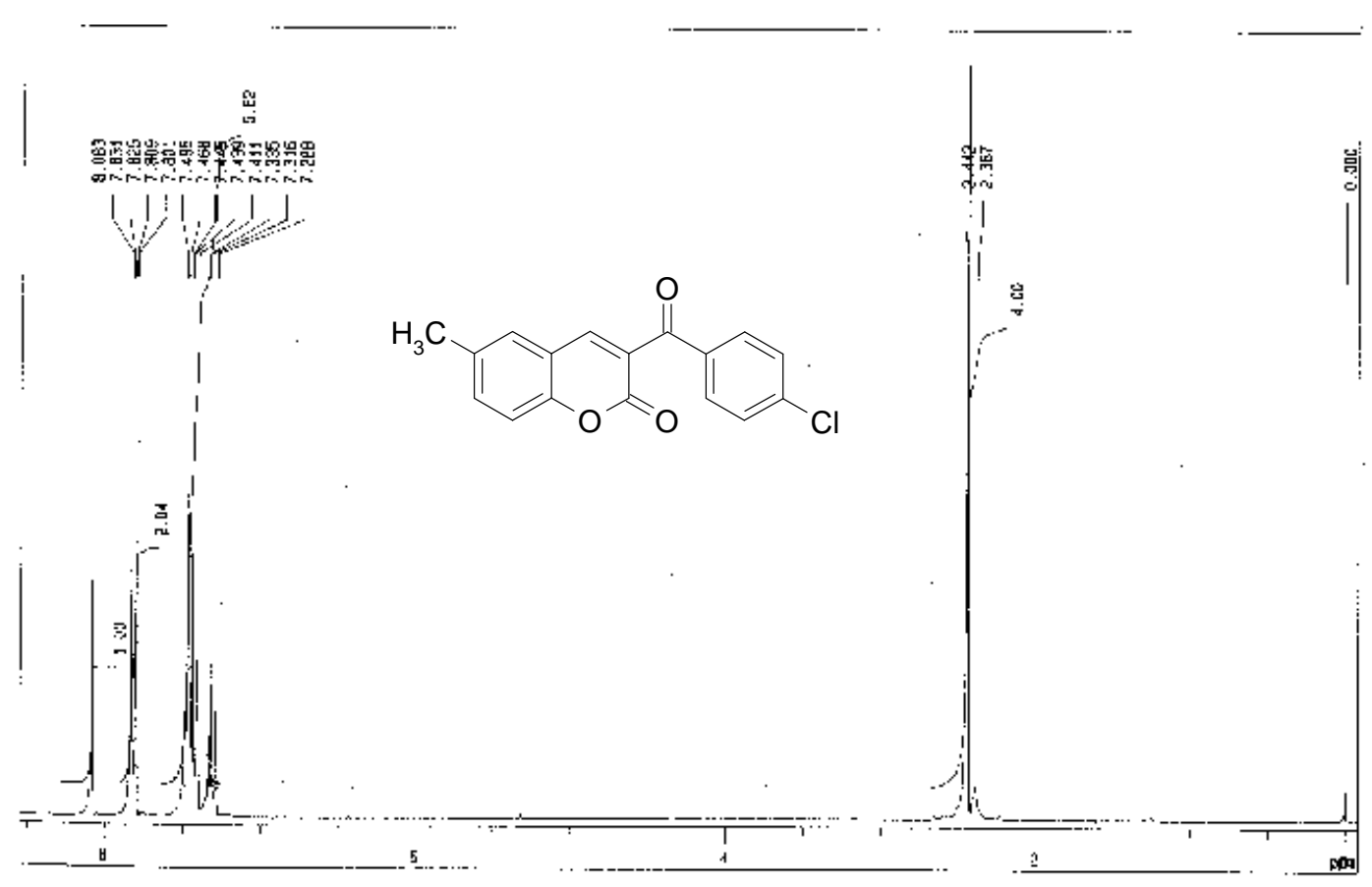

$300 \mathrm{MHz}\left(\mathrm{CDCl}_{3}\right){ }^{1} \mathrm{H}$ NMR spectrum of

3-(4-chlorobenzoyl)-6-methyl-2H-2-chromenone $\mathbf{4 f}$

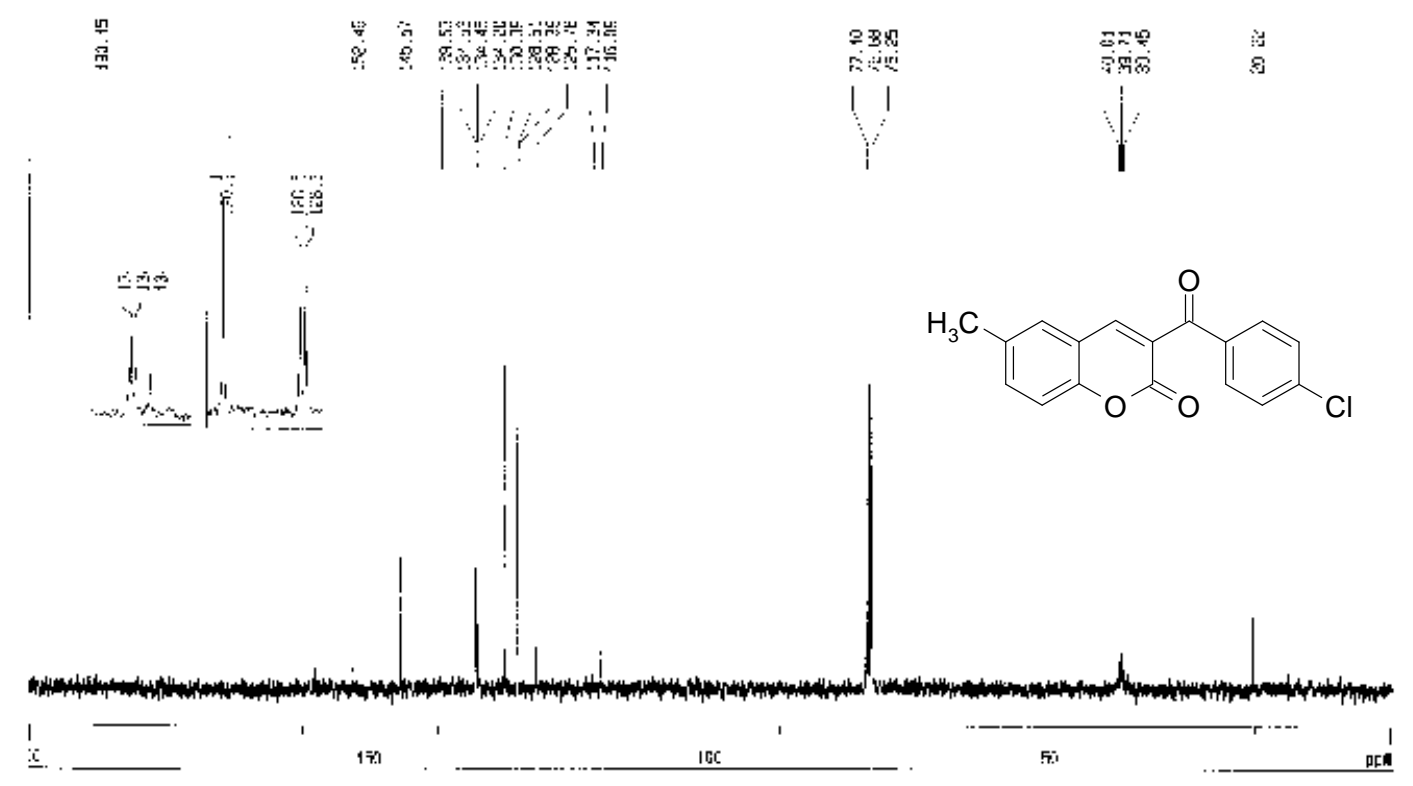

$75 \mathrm{MHz}\left(\mathrm{CDCl}_{3}\right){ }^{13} \mathrm{C}$ NMR spectrum of

3-(4-chlorobenzoyl)-6-methyl-2H-2-chromenone $\mathbf{4 f}$ 


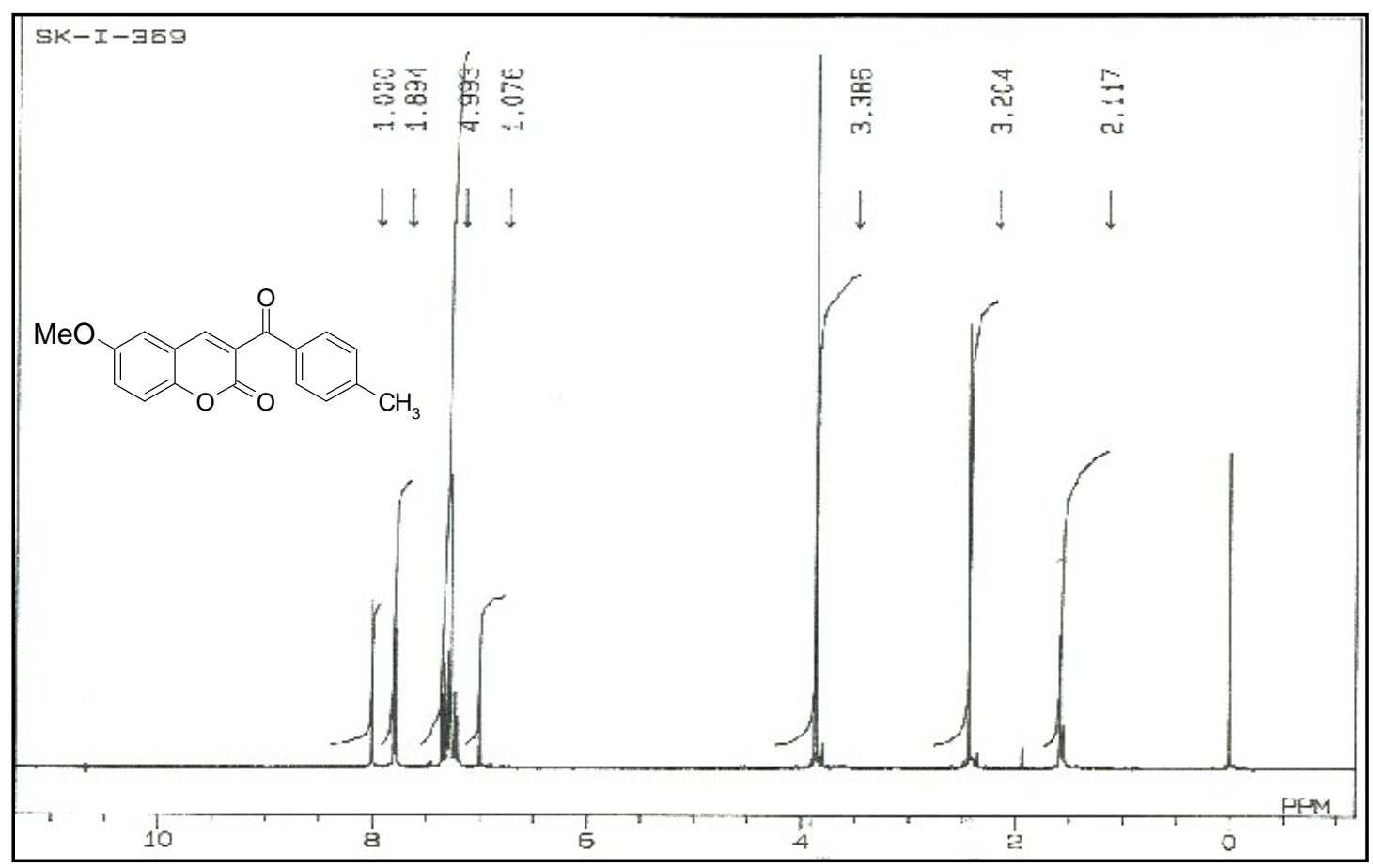

$400 \mathrm{MHz}\left(\mathrm{CDCl}_{3}\right){ }^{1} \mathrm{H} \mathrm{NMR}$ spectrum of

6-methoxy-3-(4-methylbenzoyl)-2H-2-chromenone 4 h

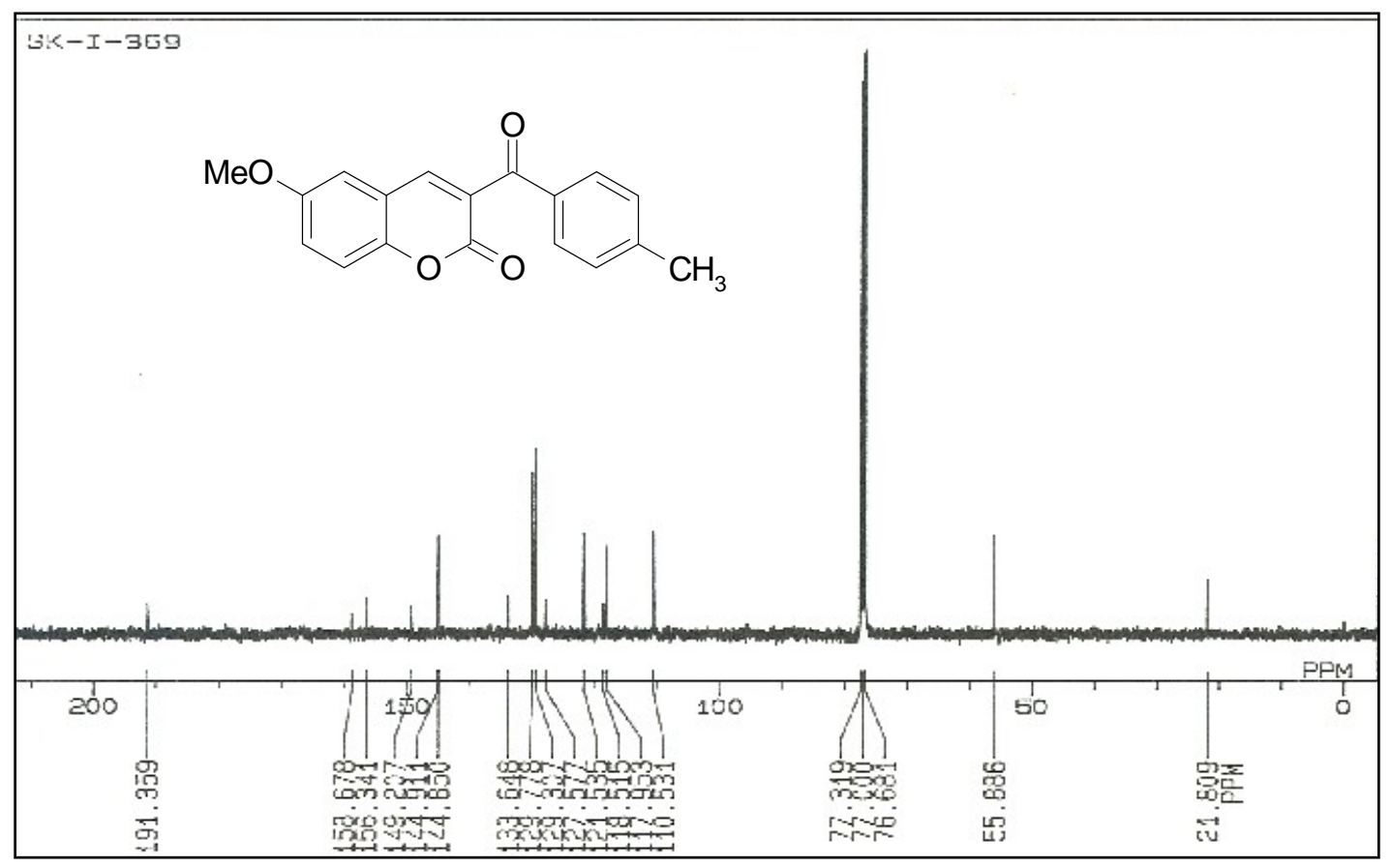

$100 \mathrm{MHz}\left(\mathrm{CDCl}_{3}\right){ }^{13} \mathrm{C}$ NMR spectrum of 6-methoxy-3-(4-methylbenzoyl)-2H-2-chromenone $\mathbf{4 h}$ 


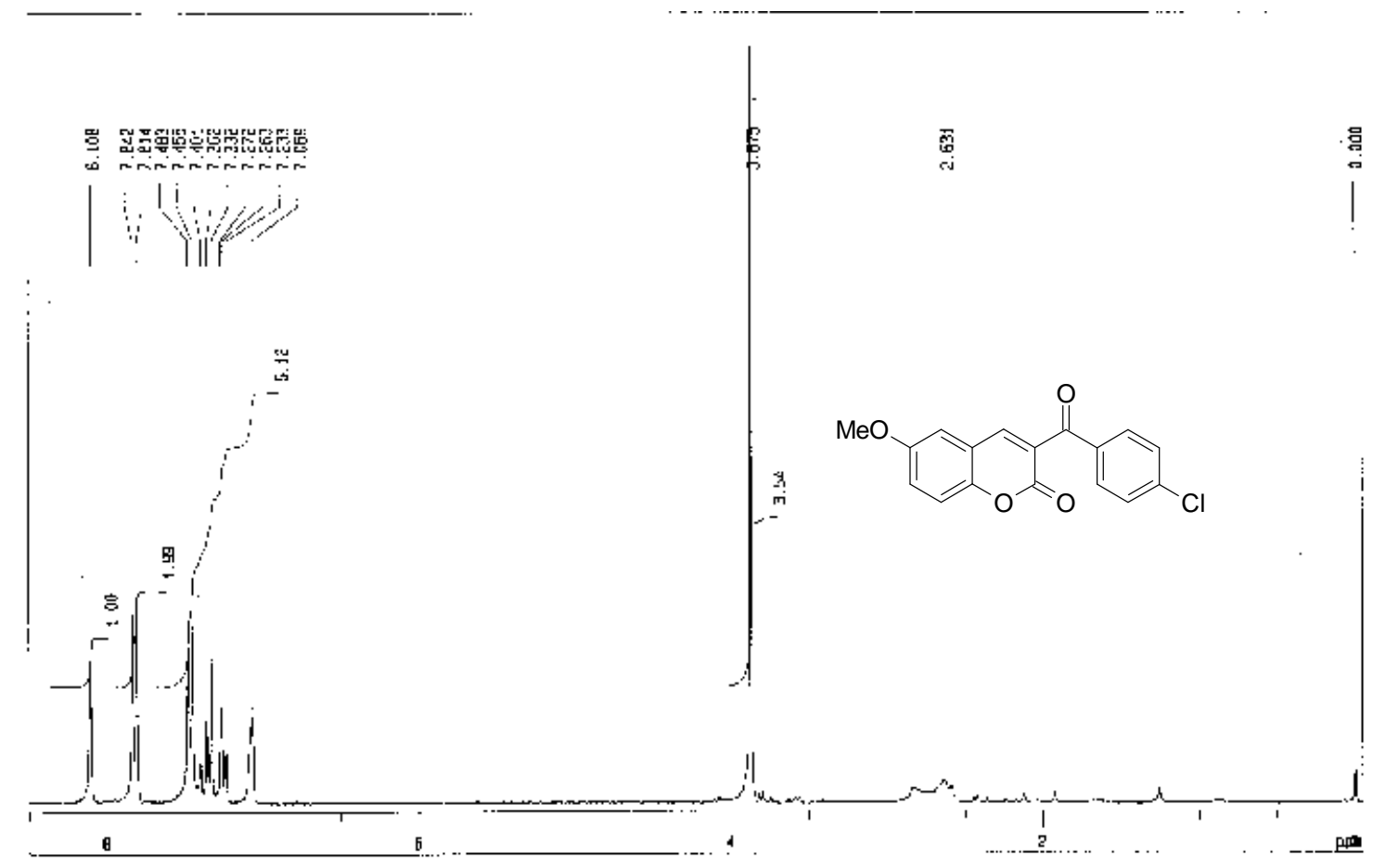

$300 \mathrm{MHz}\left(\mathrm{CDCl}_{3}+\mathrm{DMSO}\right){ }^{1} \mathrm{H} \mathrm{NMR}$ spectrum of 3-(4-chlorobenzoyl)-6-methoxy-2H-2-chromenone 4i

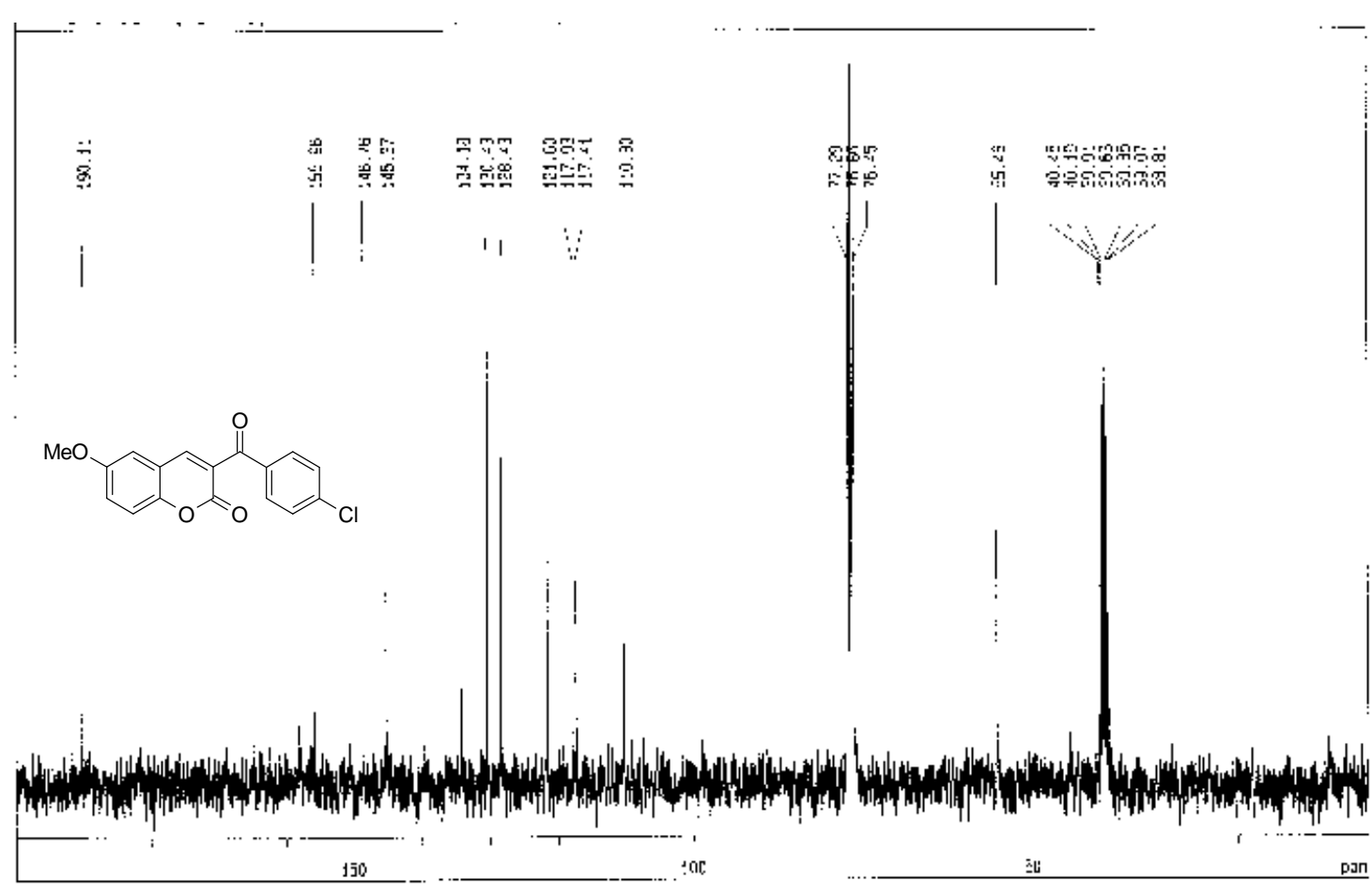

$75 \mathrm{MHz}\left(\mathrm{CDCl}_{3}+\mathrm{DMSO}\right){ }^{13} \mathrm{C}$ NMR spectrum of 3-(4-chlorobenzoyl)-6-methoxy-2H-2-chromenone 4i 


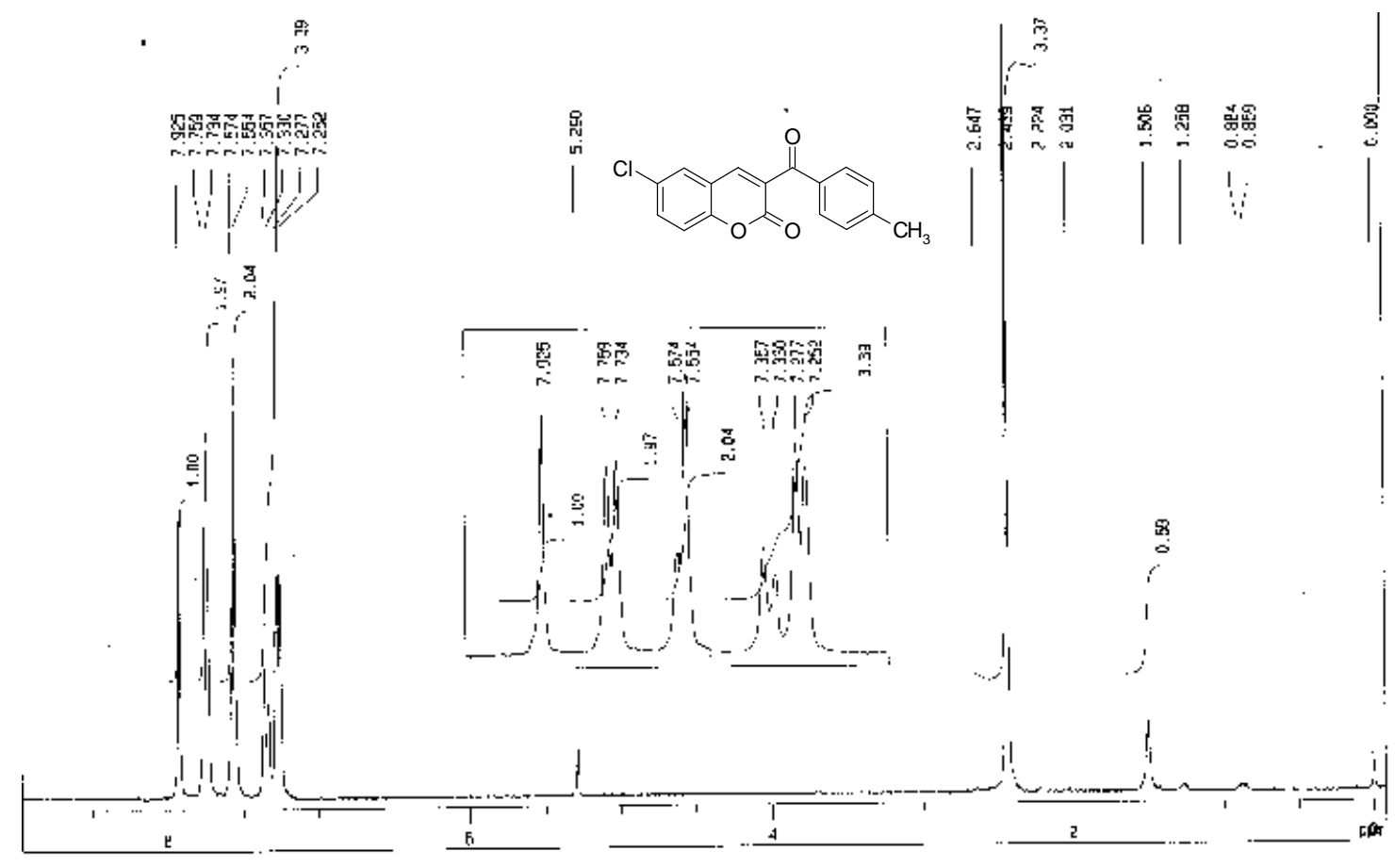

$300 \mathrm{MHz}\left(\mathrm{CDCl}_{3}\right){ }^{1} \mathrm{H}$ NMR spectrum of

6-chloro-3-(4-methylbenzoyl)-2H-2-chromenone $4 \mathbf{k}$

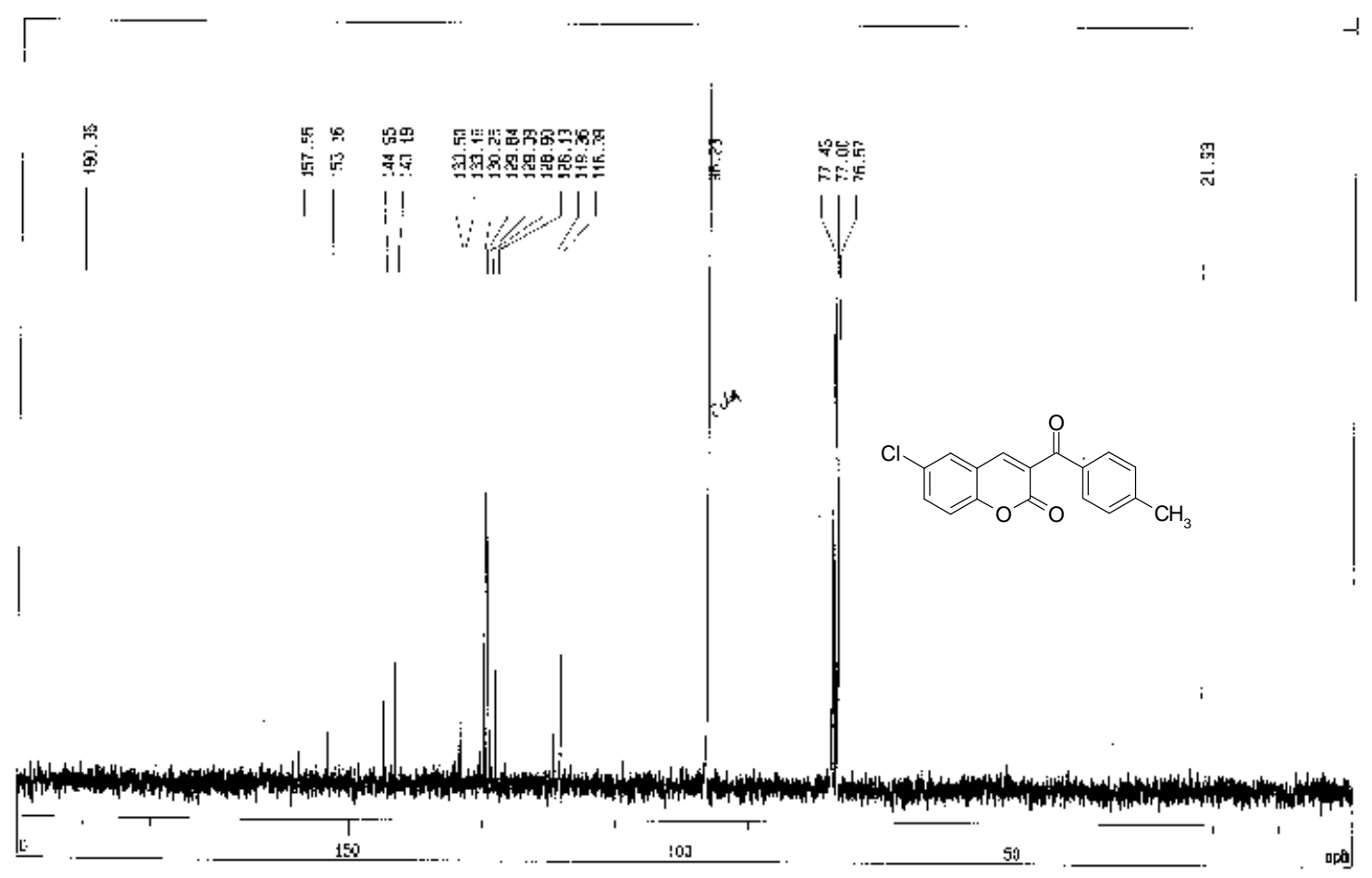

$75 \mathrm{MHz}\left(\mathrm{CDCl}_{3}\right){ }^{13} \mathrm{C} \mathrm{NMR}$ spectrum of 6-chloro-3-(4-methylbenzoyl)-2H-2-chromenone $\mathbf{4 k}$ 


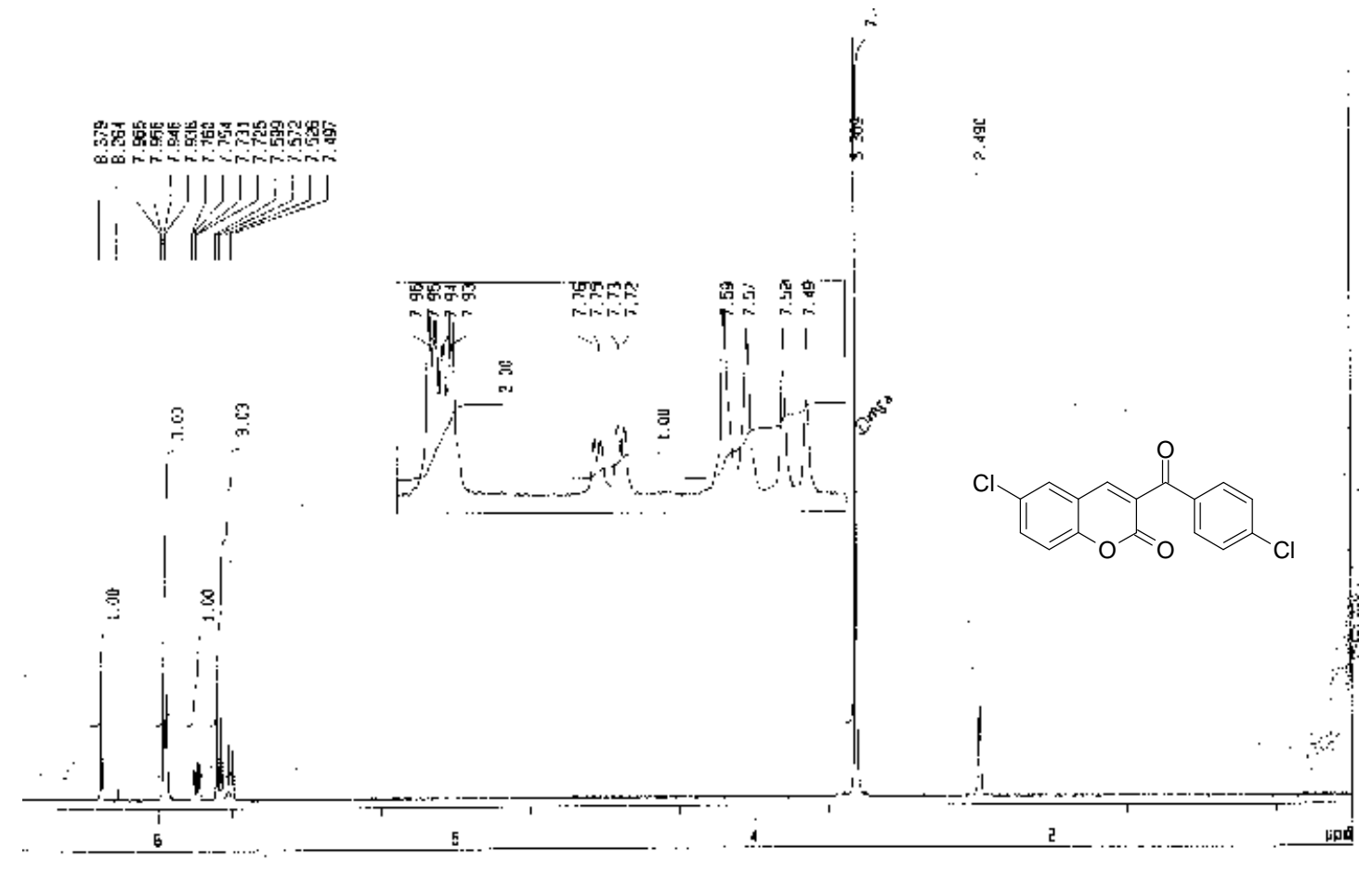

$300 \mathrm{MHz}\left(\mathrm{CDCl}_{3}\right){ }^{1} \mathrm{H}$ NMR spectrum of

6-chloro-3-(4-chlorobenzoyl)-2H-2-chromenone 41

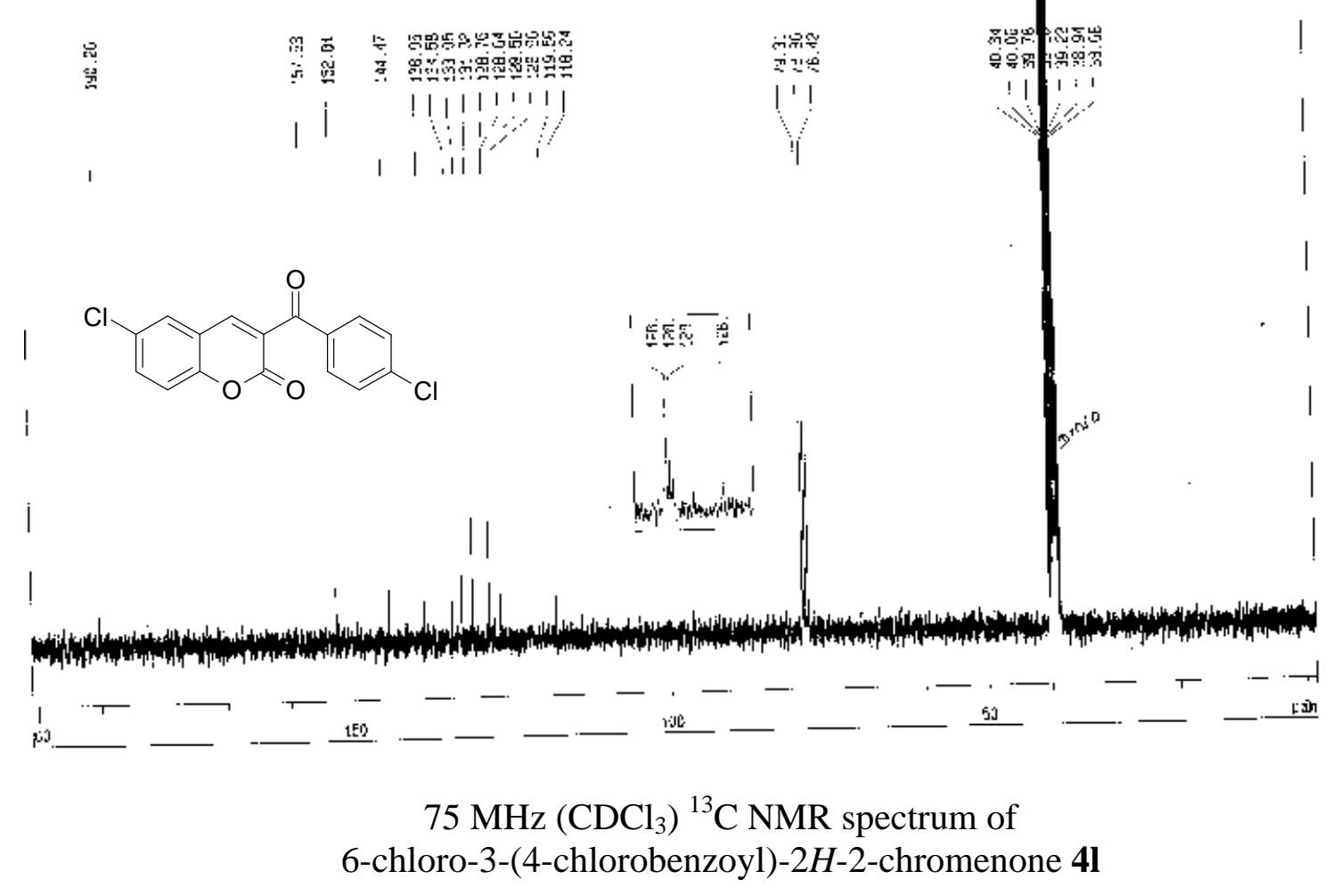




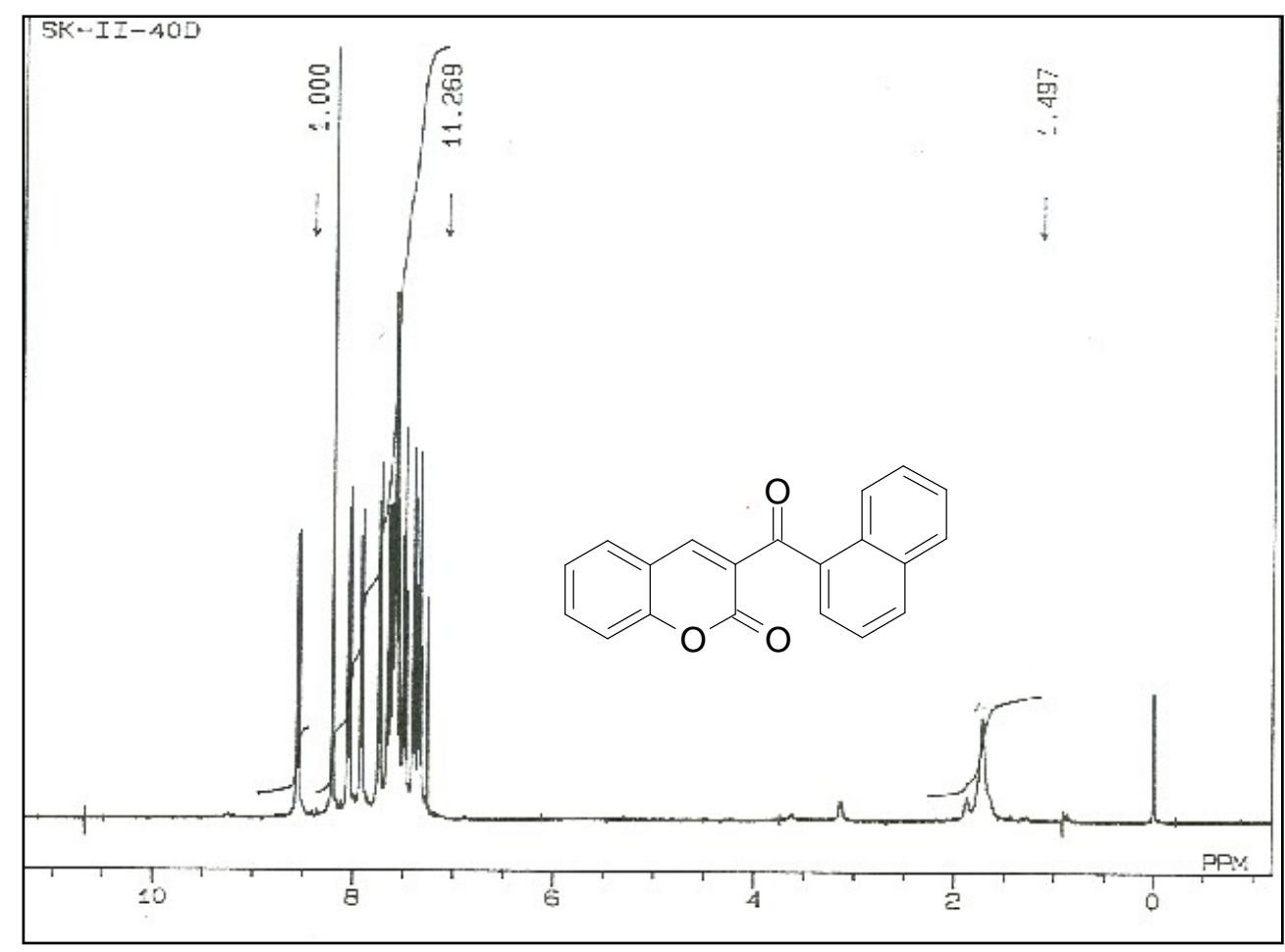

$400 \mathrm{MHz}\left(\mathrm{CDCl}_{3}\right){ }^{1} \mathrm{H}$ NMR spectrum of

3-(1-naphthylcarbonyl)-2H-2-chromenone 8

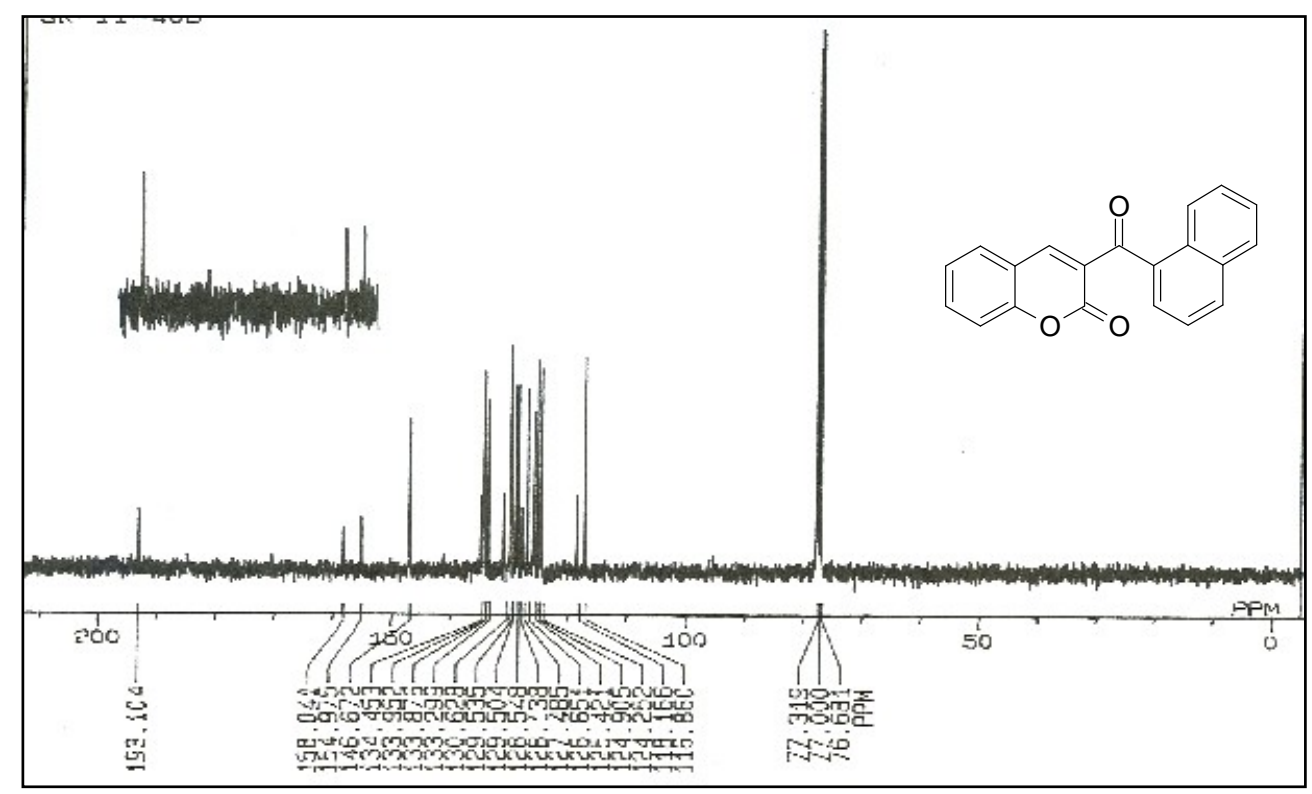

$100 \mathrm{MHz}\left(\mathrm{CDCl}_{3}\right){ }^{13} \mathrm{C} \mathrm{NMR}$ spectrum of

3-(1-naphthylcarbonyl)-2H-2-chromenone 8 


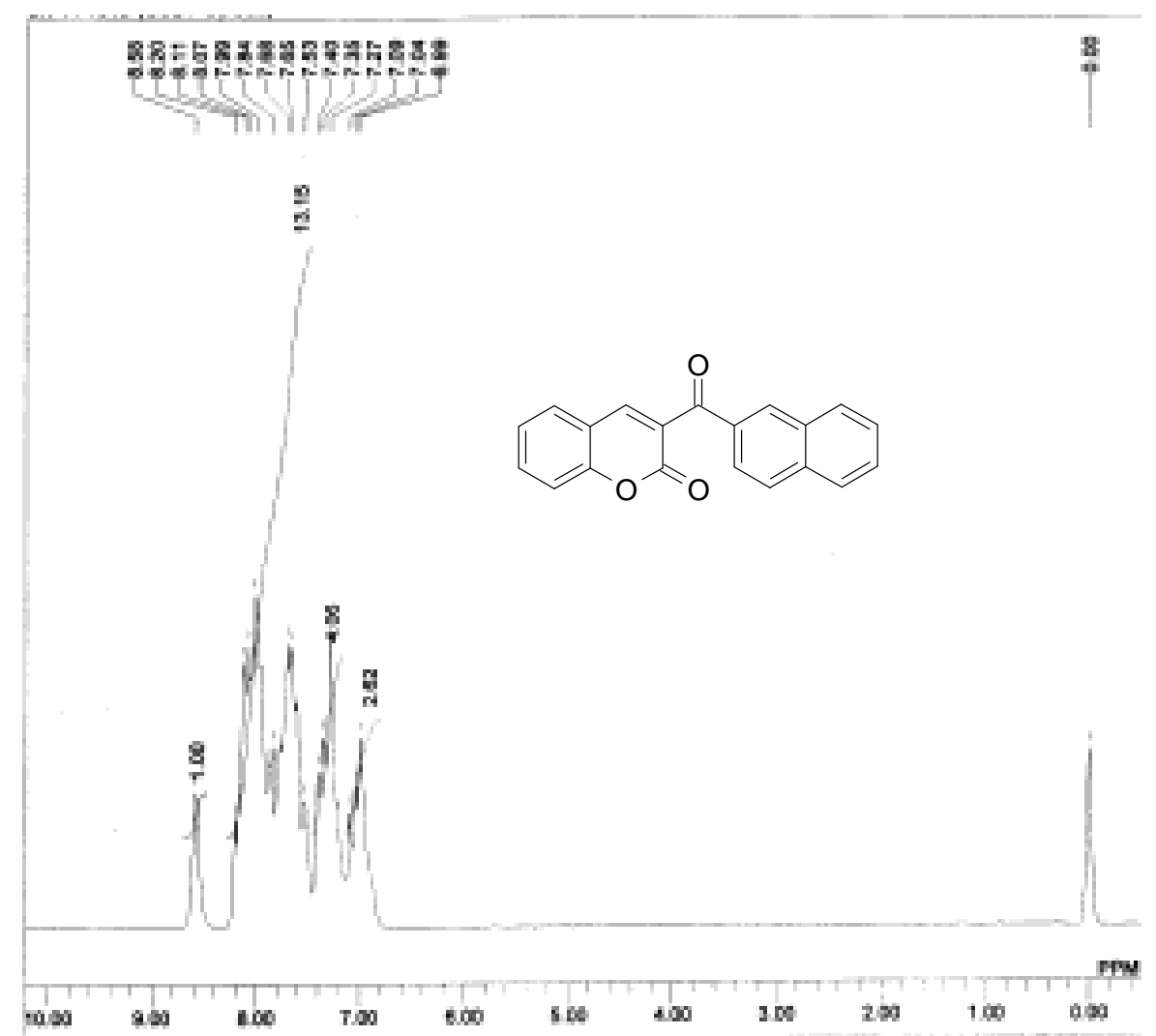

$60 \mathrm{MHz}\left(\mathrm{CDCl}_{3}\right){ }^{1} \mathrm{H}$ NMR spectrum of

3-(2-naphthylcarbonyl)-2H-2-chromenone 10

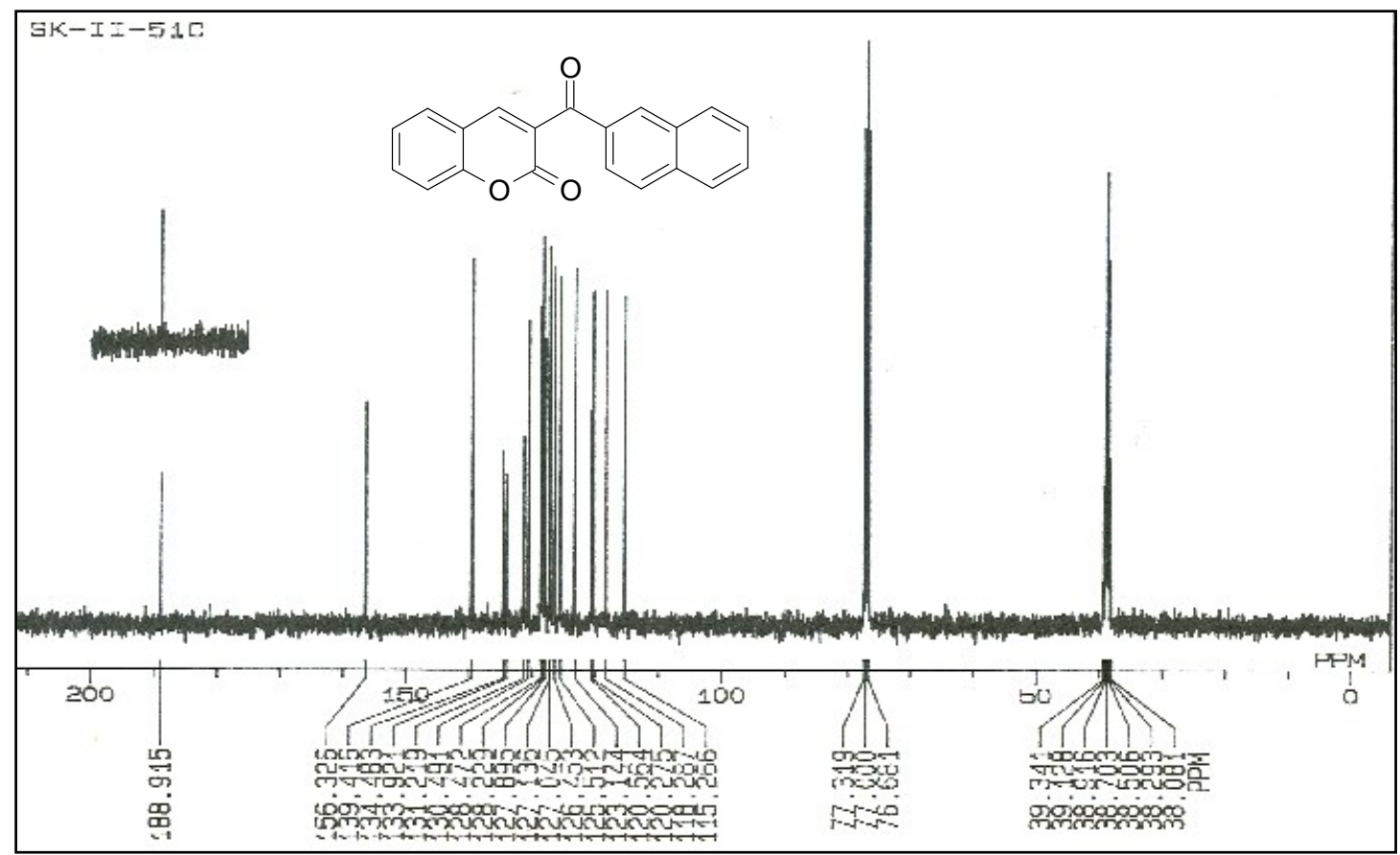

$100 \mathrm{MHz}\left(\mathrm{CDCl}_{3}+\right.$ DMSO-D 6$){ }^{13} \mathrm{C}$ NMR spectrum of

3-(2-naphthylcarbonyl)-2H-2-chromenone $\mathbf{1 0}$ 


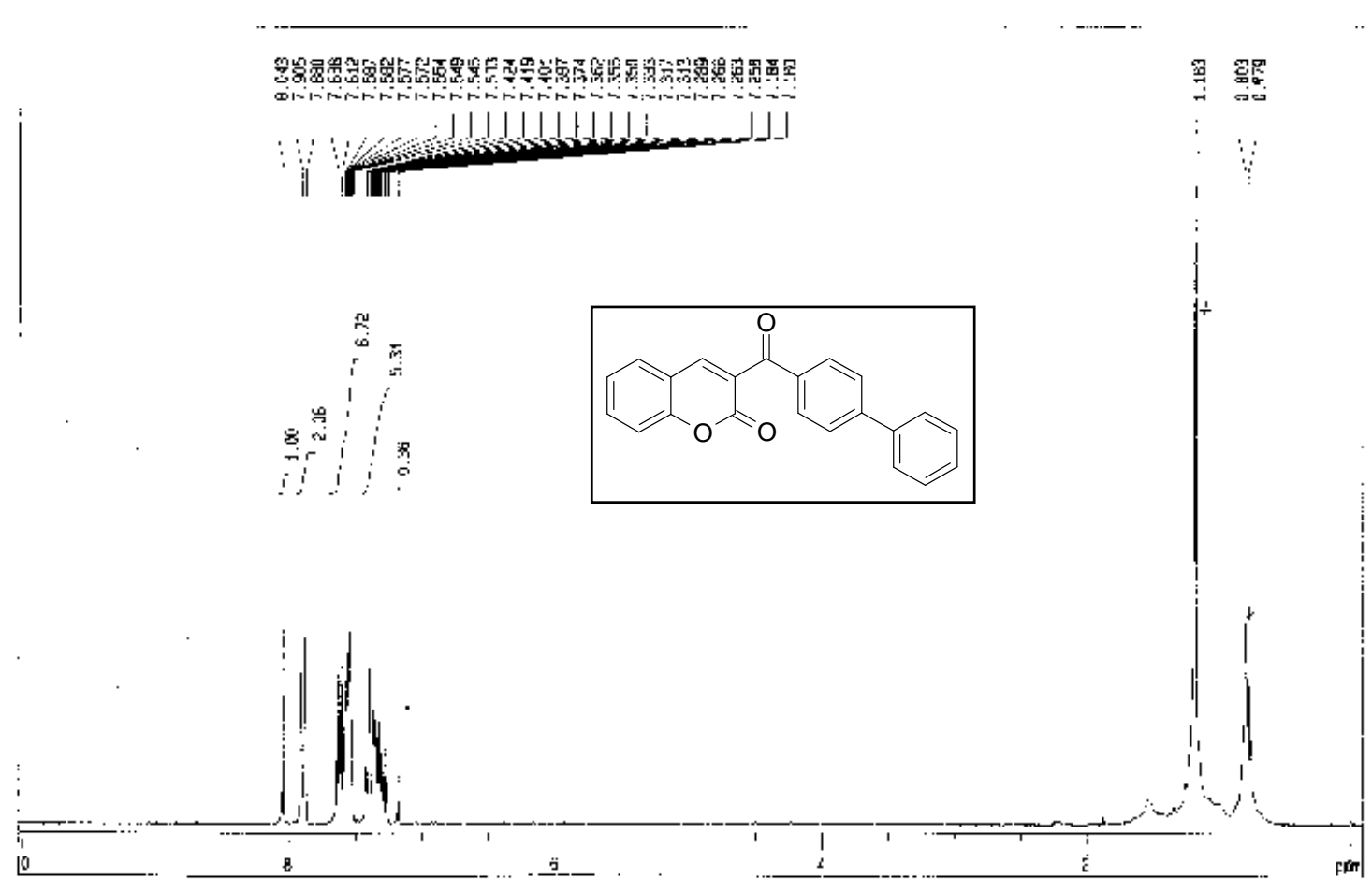

$300 \mathrm{MHz}\left(\mathrm{CDCl}_{3}\right){ }^{1} \mathrm{H}$ NMR spectrum of 3-(biphenyl-4-yl-carbonyl)-2H-2-chromenone 12

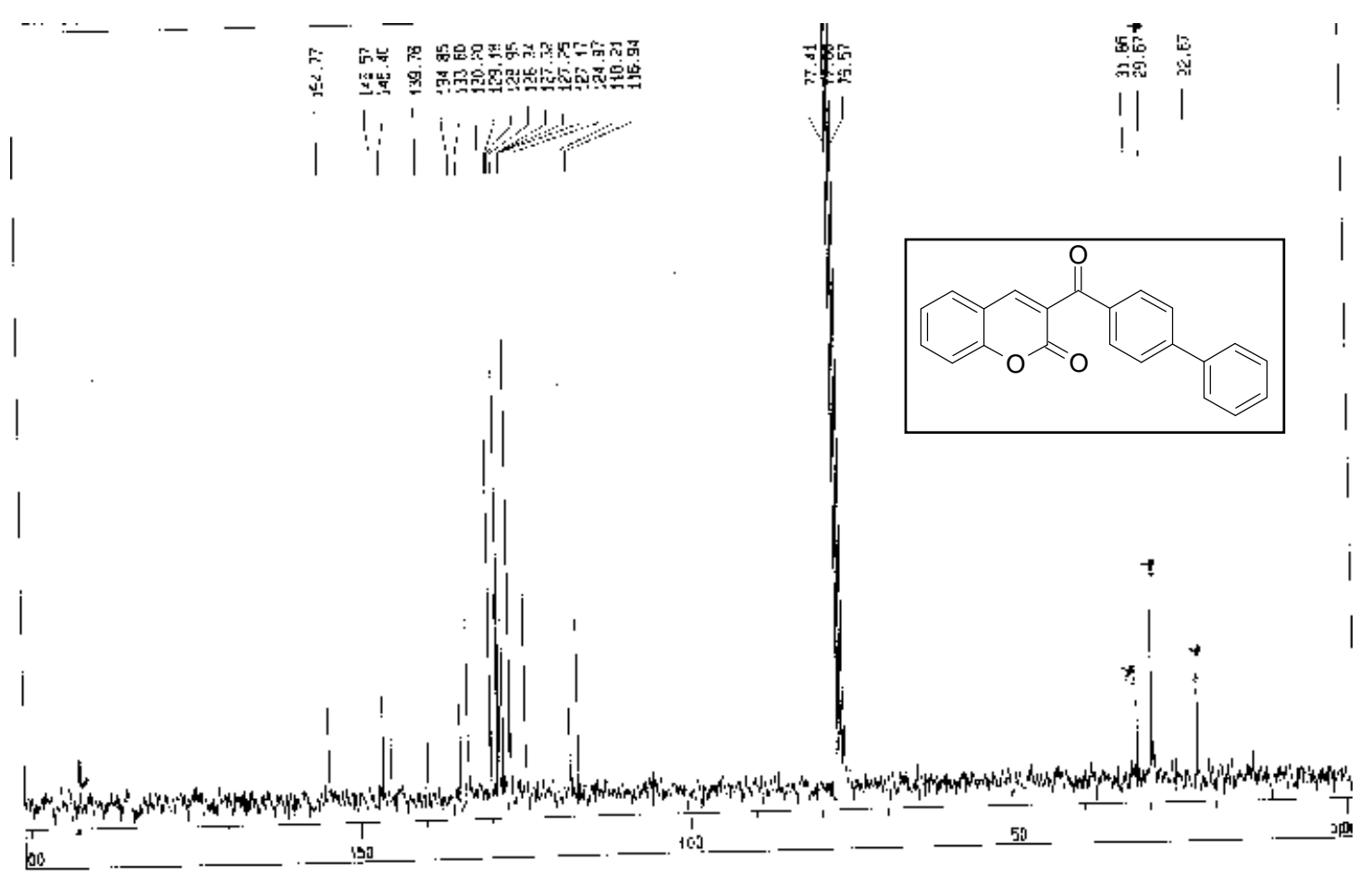

$75 \mathrm{MHz}\left(\mathrm{CDCl}_{3}\right){ }^{13} \mathrm{C}$ NMR spectrum of

3-(biphenyl-4-yl-carbonyl)-2H-2-chromenone 12 


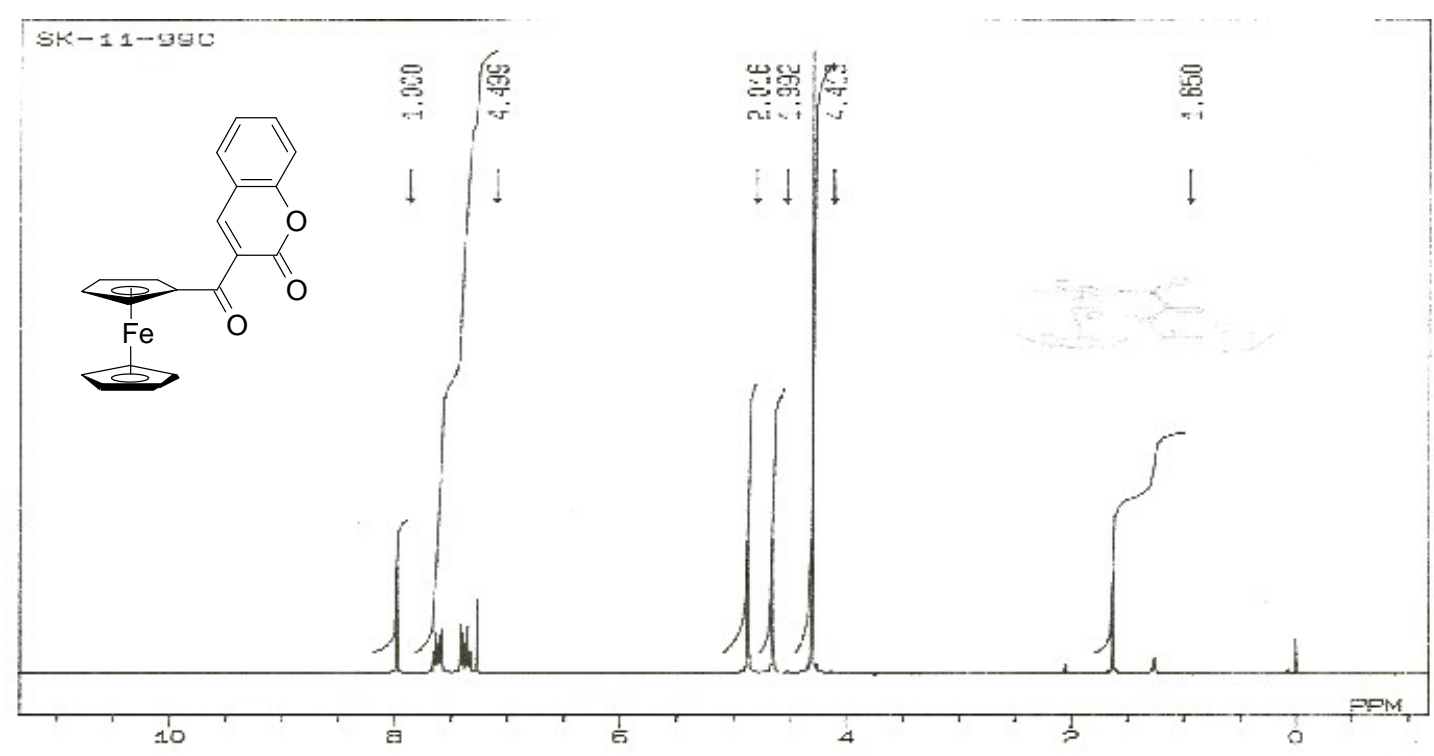

$400 \mathrm{MHz}\left(\mathrm{CDCl}_{3}\right){ }^{1} \mathrm{H}$ NMR spectrum of 3- $\eta^{5}$-ferrocenoyl-2H-2-chromenone 14

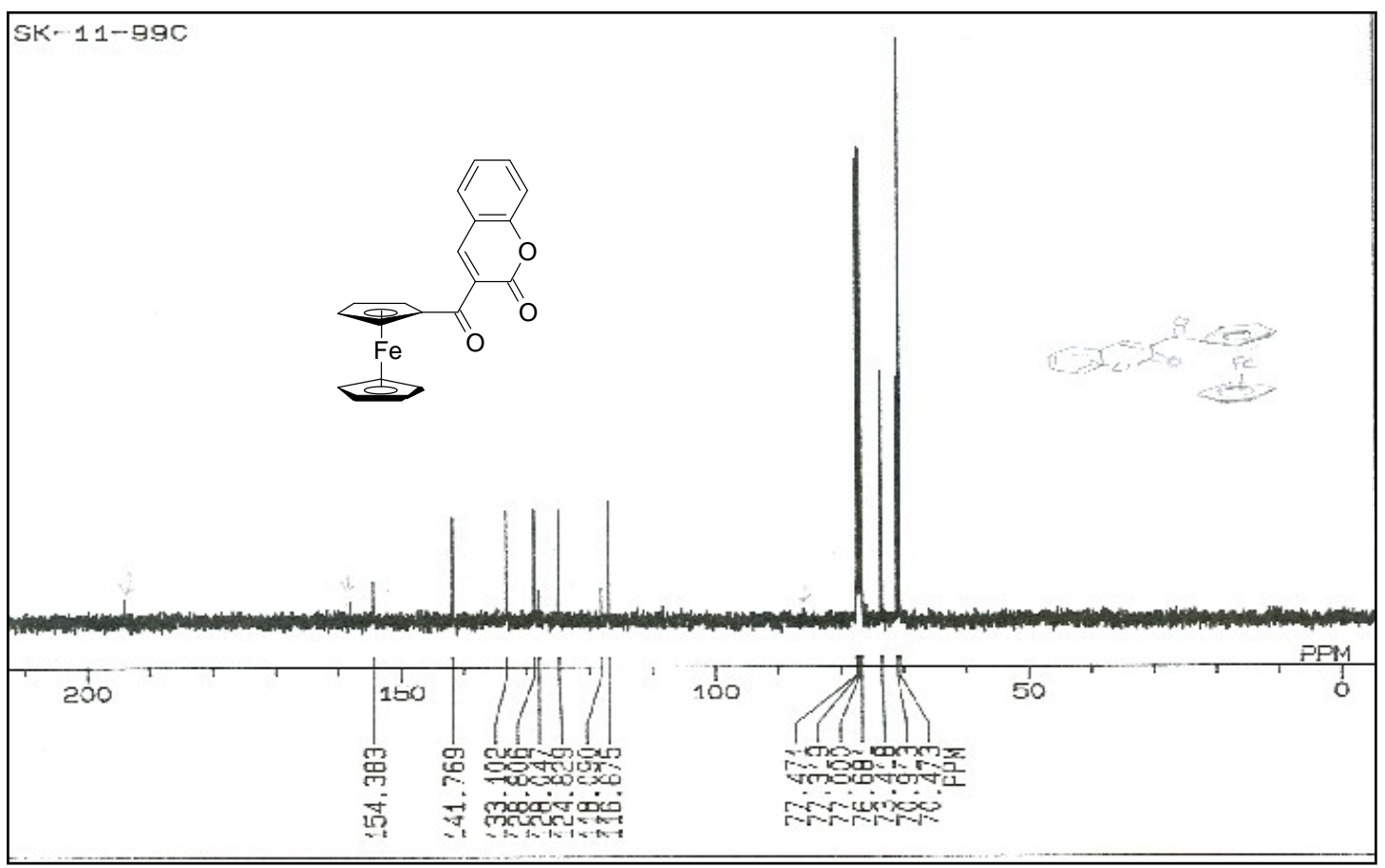

$100 \mathrm{MHz}\left(\mathrm{CDCl}_{3}\right){ }^{13} \mathrm{C}$ NMR spectrum of 3- $\eta^{5}$-ferrocenoyl-2H-2-chromenone 14 